NASA Technical Memorandum 107232

\title{
Layerwise Mechanics and Finite Element for the Dynamic Analysis of Piezoelectric Composite Plates
}

Dimitris A. Saravanos

Ohio Aerospace Institute

Brook Park, Ohio

Paul R. Heyliger

Colorado State University

Fort Collins, Colorado

and

Dale A. Hopkins

Lewis Research Center

Cleveland, Ohio

June 1996

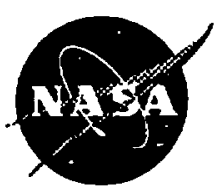

National Aeronautics and

Space Administration 
. 


\title{
LAYERWISE MECHANICS AND FINITE ELEMENT
}

\section{FOR THE DYNAMIC ANALYSIS OF PIEZOELECTRIC COMPOSITE PLATES}

Dimitris A. Saravanos ${ }^{1}$, Paul R. Heyliger ${ }^{2}$ and Dale A. Hopkins

\author{
Structural Mechanics Branch \\ NASA Lewis Research Center \\ 21000 Brookpark Rd., MS 49-8 \\ Cleveland, Ohio 44135
}

1 Senior Research Associate, Ohio Aerospace Institute.

2 Associate Professor, Civil Engineering Dept., Colorado State University 


\begin{abstract}
Laminate and structural mechanics for the analysis of laminated composite plate structures with piezoelectric actuators and sensors are presented. The theories implement layerwise representations of displacements and electric potential, and can model both the global and local electromechanical response of smart composite laminates. Finite-element formulations are developed for the quasi-static and dynamic analysis of smart composite structures containing piezoelectric layers. Comparisons with an exact solution illustrate the accuracy, robustness and capability of the developed mechanics to capture the global and local response of thin and/or thick laminated piezoelectric plates. Additional correlations and numerical applications demonstrate the unique capabilities of the mechanics in analyzing the static and free-vibration response of composite plates with distributed piezoelectric actuators and sensors.
\end{abstract}




\section{Introduction}

The development of a new class of "smart" composite materials and adaptive structures with sensory/active capabilities may further improve the performance and reliability of aeronautical structural systems. Such materials will combine the superior mechanical properties of composite materials, as well as, incorporate the additional inherent capability to sense and adapt their static and dynamic response (adaptive structures), or continuously monitor the type, location, and extent of eminent damage (health monitoring). However, this effort requires the development of admissible mechanics entailing capabilities to model the unified electromechanical response of sensory/active structures including the coupling between sensors and actuators. Additionally, the mechanics should address local through-the-thickness effects, such as the evolution of complicated stress-strain fields in smart composites and interfacial phenomena between the embedded micro-devices and passive composite plies in a smart laminate. The present paper presents the development of layerwise mechanics for the dynamic analysis of smart composite plate structures with embedded piezoelectric sensors and actuators.

There have been many theories and models proposed for the analysis of laminated composite plates containing active and passive piezoelectric layers (see e.g. Lee and Moon (1989), Lee (1990), Lee and Moon (1990), Wang and Rogers (1991), Crawley and Lazarus (1989), Tzou and Tseng (1990), Lammering (1991)). Most of these theories use simplifying approximations attempting to replicate the induced strain or electric fields generated by a piezoelectric layer under an external electric field or applied load. An exact solution by Heyliger and Saravanos (1995) for piezoelectric laminated plates has shown that the electric and elastic field distributions are often poorly modeled using simplified theories. Variational methods and finite element models for piezoelectric solids have also been reported by Allik and Hughes (1970), Naillon et al. (1983) and Ha et al. (1992). Related work has been reported for infinite piezoelectric laminates by Pauley (1974), and for finite elastic laminated beams and plates with induced strain actuation by Robbins and Reddy $(1991,1993)$. The work in this paper builds on previous layerwise laminate theories (Reddy (1987), Robbins and Reddy (1991), Robbins and Reddy (1993)), as well as, on that of the authors (Saravanos (1993), Saravanos (1994), 
Heyliger et al. (1994), Saravanos and Heyliger (1995)) to consider the complete dynamic electromechanical response of smart piezoelectric plate structures under external mechanical or electrical loading.

The paper describes layerwise laminate theories for composite structures containing embedded piezoelectric layers as sensors or actuators. According to these laminate theories, which will be called interchangeably in the remaining paper as layerwise or discrete-layer theories, each layer is modeled using independent approximations for the in-plane displacement components and the electrostatic potential in a unified representation, as mandated by the linear theory of piezoelectricity. The laminate theories assume either constant or variable transverse displacements through the laminate thickness. Finite element solutions for the dynamic response of smart structures are further developed. Application studies verify the accuracy and demonstrate the advantages and versatility of the mechanics in analyzing the dynamic response of composite plates with sensory and active piezoelectric elements.

\section{Piezoelectric Laminates}

This section describes the developed mechanics for composite laminates with embedded piezoelectric sensors and actuators. The coupled material equations for each ply are first presented in a unified way which may represent either piezoelectric or passive composite layers. Discrete-layer approximations are subsequently defined and the resultant piezoelectric laminate theories are presented. A standard laminate coordinate system is assumed, such that, $\mathrm{x}$ and $\mathrm{y}$ are the in-plane axes and the $\mathrm{z}$-direction coincides with the thickness dimension of the laminate. The general problem considered in this paper is to determine the dynamic behavior of the elastic and electric field components throughout the laminate under an applied mechanical or electrical loading. Each ply of the laminate can be composed of a purely elastic, piezoelectric, or conducting material. The forcing function may be a combination of applied tractions, electric potential, surface displacements, or charge. 


\section{Material Representation}

The constitutive equations of a piezoelectric material are given by Tiersten (1969),

$$
\begin{aligned}
& S_{i}-s_{t}^{E} \sigma_{j}+d_{d z} E_{k} \\
& D_{l}-d_{v} \sigma_{j}+e_{u}^{\sigma} E_{k}
\end{aligned}
$$

or equivalently,

$$
\begin{aligned}
& \sigma_{i}=C_{t}^{E_{j}} S_{j}-e_{a q} E_{k} \\
& D_{l}=e_{b} S_{j}+e_{u}^{S} E_{k}
\end{aligned}
$$

where: $i, j=1, \ldots, 6$ and $k, 1=1, \ldots, 3 ; \sigma_{i}$ and $S_{i}$ are the mechanical stresses and engineering strains in vectorial notation; $E_{k}$ is the electric field vector; $D_{1}$ is the electric displacement vector; $s_{i j}$ and $C_{i j}$ are the elastic compliance and stiffness tensors; $d_{\mathrm{j}}$ and $\mathrm{e}_{\mathrm{j}}$ are different forms of the piezoelectric tensor; and $\varepsilon_{1 k}$ is the electric permittivity tensor of the material. Superscripts $E, \sigma$, and $S$ indicate constant electric field, stress and strain conditions, respectively. The electric field vector $E_{k}$ is the gradient of the electric potential $\phi$

$$
E_{k}-\partial \phi / \partial x_{k}
$$

The materials are assumed to be monoclinic class 2 crystals with a diad axis parallel to the $z$ axis. The poling direction of the material is assumed coincident with the $\mathrm{z}$ axis. The assumed material class is general enough, such that eqs. (1) or (2) may encompass the behavior of off-axis homogenized fibrous piezoelectric plies, as well as, passive composite plies [e] $=0$.

The energy stored (electric enthalpy) in the piezoelectric layer includes the components of elastic strain energy, piezoelectric energy and electric energy (Tiersten (1969)),

$$
H_{l}-\frac{1}{2}\left(C_{t} S_{l} S_{j}-2 e_{l} E_{l} S_{j}-\epsilon_{l} E_{E} E_{l}\right)
$$




\section{Discrete-Layer Laminate Theory}

Two new layerwise laminate theories (also called discrete-layer theories) are proposed in this paper using piecewise continuous approximations along the z-axis for both displacement and electric potential fields. Previous work has demonstrated the advantages of layerwise approaches in capturing local intralaminar and interlaminar effects in elastic (Reddy (1987)) and elastodynamic problems (Saravanos (1993), Saravanos (1994)) of composite plates. Consequently, the suitability of discretelayer theories to represent the additional heterogeneity induced by the presence of embedded piezoelectric sensors/actuators in composite laminates can not be understated.

In the present approach, the electric potential is included in the state variables. The laminate is subdivided in N-1 sublaminates (or discrete layers), and continuous fields are assumed in each sublaminate, such that, piecewise continuous representations result through the thickness of the laminate (see Fig. 1) of the following form:

$$
\begin{aligned}
& u(x, y, z, t)-\sum_{j=1}^{N} u^{J}(x, y, t) \Psi^{J}(z) \\
& v(x, y, z, t)-\sum_{j=1}^{N} v^{J}(x, y, t) \Psi^{J}(z) \\
& w(x, y, z, t)-\sum_{j=1}^{N} w^{J}(x, y, t) \Psi^{w^{J}}(z) \\
& \phi(x, y, z, t)-\sum_{j=1}^{N} \phi^{\prime}(x, y, t) \Psi^{J}(z)
\end{aligned}
$$

where $u, v, w$ are the displacements along the $x, y$ and $z$ axes; superscript $j$ indicates the points $z^{j}$ at the beginning and end of each discrete layer, $u^{j}, v^{j}, w^{j}, \phi^{j}$ are the respective displacements and electric potential at each point $z^{j}$ (see Fig. 1); $\Psi^{j}(z)$ are interpolation functions. Two unique advantages of the method are obvious: (1) the complete electromechanical state of the smart laminate is represented; and (2) the formulation entails the inherent option to select the detail of approximation in both electric and displacement fields. At the lower limit of one discrete-layer $(\mathrm{N}=2)$ the method reduces to "singlelayer" type of laminate theories, and for linear $\Psi(z)$ it reduces to the first-order shear theory. Moreover, the through the thickness displacement can be assumed constant $\left(\Psi^{w}(z)=1\right)$ or variable, 
which establishes the difference between the two laminate theories. The first, which will be identified as the w-constant theory, results by assuming $\Psi^{\prime \prime}(z)=1$ in eqs. (5) and is effectively a plate laminate theory. The second, which is identified as the w-variable theory, is a three-dimensional laminate theory. In the present paper only linear interpolation functions $\Psi(z)$ were considered.

In the context of eqs. (5), the engineering strain and electric field components become,

$$
\begin{aligned}
& S_{(x, y, z, t)}=\sum_{\substack{j=1 \\
N}}^{N} S_{1}^{f}(x, y, t) \Psi^{J}(z) \quad i=1,2,6 \\
& s_{3}(x, y, z, t)-\sum_{j=1}^{N} s_{3}^{j}(x, y, x) \Psi_{3}^{w j}(z) \\
& S_{f}(x, y, z, t)-\sum_{j=1}^{N} S_{i}^{j}(x, y, t) \Psi_{,(z)+S_{(}^{J}}^{J}(x, y, t) \Psi^{w /(z)} \quad i-4,5 \\
& E(x, y, z, t)=\sum_{j=1}^{N} E_{i}^{f}(x, y, t) \Psi^{f}(z) \quad i=1,2 \\
& E_{3}(x, y, z, t)-\sum_{j=1}^{N} E_{3}^{j}(x, y, t) \Psi_{,}^{j}(z)
\end{aligned}
$$

where the $\left\{S^{j}\right\}$ and $\left\{E^{j}\right\}$ are respectively the generalized strain and electric field vectors defined as follows:

$$
\begin{aligned}
& s_{1}^{j}=U_{x}^{j} \quad s_{2}^{j}-V_{y}^{j} \quad s_{6}^{j}-U_{y^{*}}^{j} V_{x}^{j} \quad s_{3}^{J}-W^{j} \quad s_{4}^{j}-V^{j} \quad s_{5}^{j}=U^{j} \\
& \vec{S}_{4}^{\prime}-W_{y}^{j} \quad S_{5}^{J} W_{x}^{j} \\
& E_{1}^{J}-\Phi_{x}^{J} \quad E_{2}^{J}-\Phi_{y}^{J} \quad E_{3}^{J}-\Phi^{J}
\end{aligned}
$$

The electric enthalpy of the laminate is by definition,

$$
H_{L^{-}} \int_{-W_{2}}^{M / 2} H_{l} d
$$

where $h$ is the laminate thickness. By combining eqs. $(4,6,8)$ and integrating through-the-thickness, the energy stored in a piezoelectric laminate is obtained as a quadratic expression of the generalized strain/electric field and the generalized laminate stiffness, piezoelectric and dielectric permittivity matrices, 


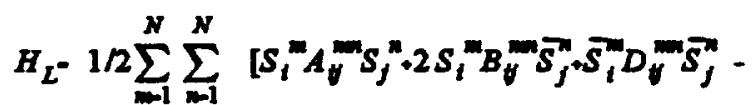

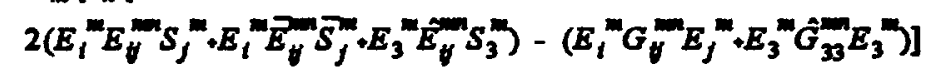

In the above equation, $A^{m m}, B^{m n}$, and $D^{m n}$ are the generalized laminate stiffness matrices,

$$
\begin{aligned}
& A_{t j}^{m} \cdot \sum_{L 1}^{L} \int_{z_{1}}^{z h} C_{t j} \Psi^{m}(z) \Psi^{n}(z) d z \quad i, j-1,2,6
\end{aligned}
$$

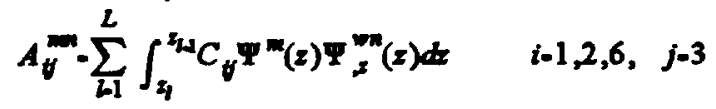

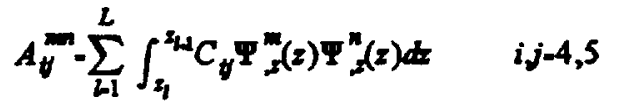

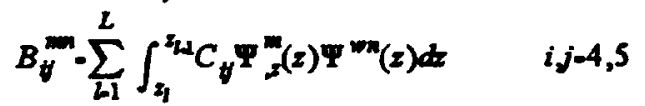

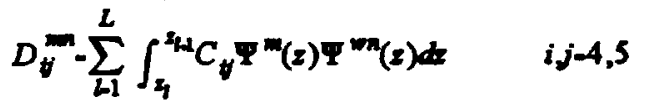

$\mathrm{E}^{\mathrm{mn}}$ are the piezoelectric laminate matrices,

$$
\begin{aligned}
& E_{\forall}^{m}-\sum_{L 1}^{L} \int_{z_{1}}^{z_{11}} e_{\psi^{\prime}} \Psi^{m}(z) \Psi_{2}^{n}(z) d z \quad i-3 \quad j=1,2,6
\end{aligned}
$$

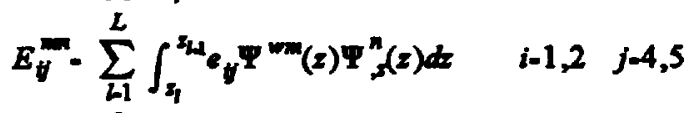

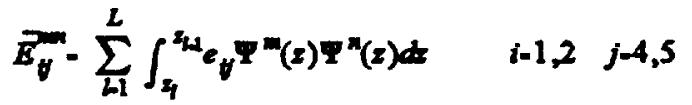

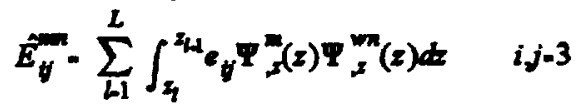

and $\mathrm{G}^{\mathrm{mm}}$ are the laminate matrices of electric permittivity,

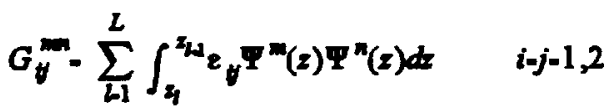

$$
\begin{aligned}
& G_{t}^{m-}-\sum_{L-1}^{L} \int_{z_{1}}^{x_{L} \varepsilon_{t}} \Psi_{g}^{m}(z) \Psi_{2}^{n}(z) d t \quad i-j-3
\end{aligned}
$$

where $\mathbf{L}$ is the number of plies in the laminate. 


\section{Piezoelectric Structures}

The starting point for the equations of motion of a composite structure with embedded active/sensory piezoelectric layers in a variational form, is the Hamilton's principle for a piezoelectric medium (Tiersten (1969))

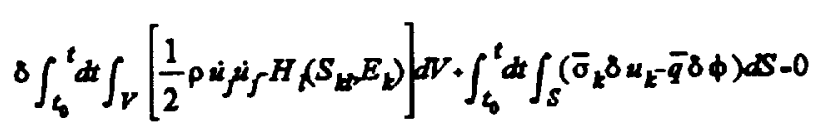

Here $t$ is time, $V$ and $S$ are the volume and surface occupied by and bounding the solid, overbar $\sigma$ and overbar $q$ are the surface tractions and surface charge, respectively, $\delta$ is the variational operator, the overdot represents differentiation with respect to time, and $\mathrm{H}_{1}$ represents the local electric enthalpy.

Integration through-the-thickness yields the Hamilton's principle (equations of motion) in terms of the generalized laminate quantities defined in the previous section

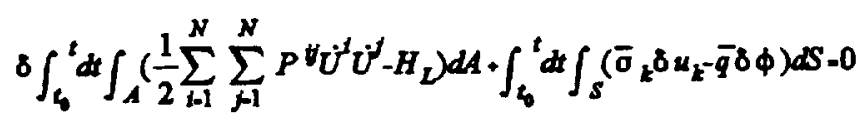

where, $A$ is the mid-plane area occupied by the plate, $H_{L}$ is the electric enthalpy of the laminate given by eq. (9), and $\mathrm{P}^{\mathrm{ij}}$ are the generalized densities (per unit area) of the laminate given by

$$
P^{t} \sum_{l-1}^{L} \int_{z_{l}}^{z_{L 1}} P_{l} \Psi^{I}(z) \Psi^{J}(z) d z
$$

To develop structural solutions, local in-plane approximations of the generalized electromechanical state (displacements and electric potential) in eq. (5) are proposed of the following type. 


$$
\begin{aligned}
& U^{J}(x, y, t)=\sum_{i=1}^{M} U^{\prime \prime}(t) R_{i}^{*}(x, y) \\
& V^{J}(x, y, t)-\sum_{i=1}^{M} V^{\prime \prime}(t) R_{i}^{y}(x, y) \\
& W^{J}(x, y, t)-\sum_{i=1}^{M} W^{\prime \prime}(t) R_{i}^{w}(x, y) \\
& \Phi^{\prime}(x, y, t)-\sum_{i=1}^{M} \Phi^{\prime \prime}(t) R_{i}^{\phi}(x, y)
\end{aligned}
$$

where $U^{j}$ is the value of the generalized displacement component $\dot{U}$ corresponding to the $i$-th in-plane interpolation function $R_{i}(x, y)$, and so forth. This formulation forms the basis of the finite element technique, which is subsequently described.

\section{Finite Element Formulation}

For structural problems with general boundary, geometry and material configurations, the local inplane approximations in eq. (16) are used to develop finite element based solutions. Substituting these approximations into the equation of motion (14) and collecting the coefficients, allows the governing dynamic equations of the structure to be expressed in a discrete matrix form as,

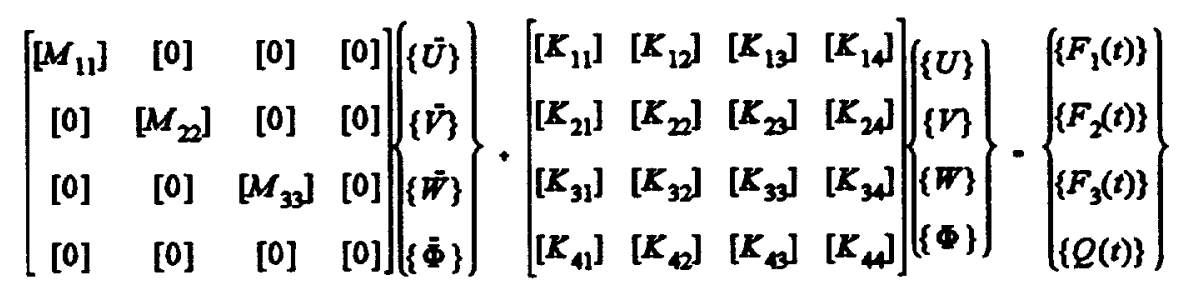

The elements of these matrices are calculated from the generalized laminate matrices defined in eqs. (10-12) as determined by the variational statement. The nature of the submatrices depends also on the approximation used for $w(z)$. For variable $w$, the structure of $\left[\mathrm{K}^{\mathrm{j} 3}\right]$ is similar to those of the other matrices. For constant $w$, the submatrices within $\left[K^{13}\right],\left[K^{23}\right]$, and $\left[K^{43}\right]$ are column vectors and those in $\left[\mathrm{K}^{33}\right]$ become scalars. In general, the submatrices within each $\left[\mathrm{K}^{\mathrm{ij}}\right]$ are each of order $(\mathrm{N})$, while the matrices $\left[\mathrm{K}^{\mathrm{ij}}\right]$ depend on the order of the in-plane approximation (M). 
Based on the above formulation, a 4-node finite element was developed with linear in-plane shape functions. Reduced integration (single point Gauss quadrature) was implemented in the calculation of the shear stiffness terms to eliminate overstiffening at low thickness. The final representation of the coupled dynamic system can be expressed in fairly compact form:

$$
\left[\begin{array}{cc}
{\left[M_{m u}\right]} & 0 \\
0 & 0
\end{array}\right]\left\{\begin{array}{c}
\{\bar{U}\} \\
\{\bar{\phi}\}
\end{array}\right\} \cdot\left[\begin{array}{ll}
{\left[K_{w}\right]} & {\left[K_{w \downarrow}\right.} \\
{\left[K_{\phi}\right]} & {\left[K_{\psi \phi}\right.}
\end{array}\right]\left\{\begin{array}{l}
\{U\} \\
\{\phi\}
\end{array}\right\}\left\{\begin{array}{c}
\{F(t)\} \\
\{Q(t)\}
\end{array}\right\}
$$

Assuming that both sensory and active piezoelectric layers are embedded into the structure, the electric potential vector is subdivided in a free or sensory component $\phi^{\mathrm{F}}$ representing the voltage output at the sensors, and a forced or active component $\phi^{\wedge}$ representing the voltage imposed on the active piezoelectric layers, such that $\{\phi\}=\left\{\phi^{\mathrm{F}} ; \phi^{\mathrm{A}}\right\}$. Separating the active and sensory potential components in eq. (18), the equations take the following form

$$
\left[\begin{array}{cc}
{\left[M_{w u}\right]} & 0 \\
0 & 0
\end{array}\right]\left\{\begin{array}{c}
\{\bar{u}\} \\
\left\{\bar{\phi}^{F}\right\}
\end{array}\right\}+\left[\begin{array}{cc}
{\left[K_{w u}\right]} & {\left[K_{w \phi}^{F P}\right]} \\
{\left[K_{\psi k}^{F P}\right]} & {\left[K_{\psi \phi}^{F P}\right]}
\end{array}\right]\left\{\begin{array}{c}
\{u\} \\
\left\{\phi^{F}\right\}
\end{array}\right\}\left\{\begin{array}{c}
\{F(t)\}-\left[K_{u \phi}^{F A}\right]\left\{\phi^{A}\right\} \\
\left\{Q^{P}(t)\right\}-\left[K_{\phi \phi}^{F A}\right]\left\{\phi^{A}\right\}
\end{array}\right\}
$$

where superscripts $F$ and $A$ indicate the partitioned submatrices in accordance with the selected sensory and active configuration, respectively. The left-hand side includes the unknown electromechanical response of the structure $\left\{u, \phi^{F}\right\}$, that is, the resultant displacements and voltage at the sensors. The right-hand includes the excitation of the structure in terms of mechanical loads and applied voltages on the actuators. The electric charge at the sensors $Q^{F}(t)$ remains constant with time (practically open-circuit conditions) and is assumed equal to zero.

Among the obvious advantages is the capability of the mechanics to model the response of the piezoelectric structure either: in "active" mode, that is, with specified voltages $\Delta \phi^{\wedge}$ applied across the piezoelectric layers to induce a desirable deflection/strain state; or in "sensory" mode where displacements or mechanical loads are applied to the structure and the resultant voltage or charge is monitored; or in combined "active/sensory" mode.

Additional manipulation of eqs. (19) results in the following uncoupled dynamic equations for the 
structural displacements and sensory voltages respectively

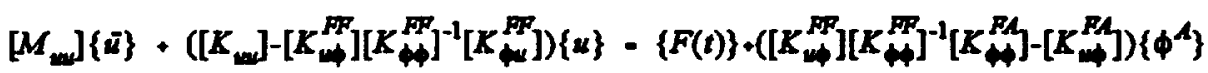

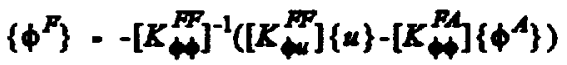

Further inspection of eq. (20) reveals that the "induced-strain" approaches, in the presence of sensors, neglect the coupling effects on both the stiffness and the induced piezoelectric force. The above dynamic system may be solved to obtain, either the modal characteristics (free-vibration), or the forced frequency response or the transient response of the piezoelectric plate.

\section{Evaluations and Discussion}

Application cases on laminated composite plates with active and sensory piezoceramic layers are presented in this section. The free-vibration response of a simply supported cross-ply plate with surface bonded continuous piezoceramic layers is analyzed first. Additional evaluations are presented on the active quasi-static response and free-vibration response of laminated cantilever plates with distributed discrete piezoelectric patches. When applicable, correlations with published data are presented. In all cases, the standard laminate notation is augmented to indicate the lamination and the location of the piezoelectric material through the thickness, with the letter $p$ indicating the piezoelectric layer.

\section{Hybrid Simply-Supported Plates.}

The free-vibration response of a 5 -ply [p/0/90/0/p] laminated simply-supported square plate incorporating piezoelectric layers and composite plies was analyzed. Two thickness aspect ratios are considered corresponding to a thin square plate $(\alpha / \mathrm{h}=50)$ and a thick plate $(\alpha / \mathrm{h}=4)$, where $\alpha$ is the free span and $h$ is the plate thickness, to illustrate the range of application of the mechanics. Results obtained with the finite-element models are compared with an exact piezoelasticity solution reported 
by Heyliger and Saravanos (1995). The laminate configuration consists of a [0/90/0] Gr/Epoxy cross-ply sublaminate with composite plies each $0.267 \mathrm{~h}$ thick, where $\mathrm{h}$ is the plate thickness. Two continuous PZT-4 layers of thickness $0.1 \mathrm{~h}$ each are also bonded to the upper and lower surfaces of the laminate. The elastic, piezoelectric and dielectric properties of the laminate materials are shown in Table 1. To comply with the reported results of the exact solution, all layers were assumed to have equal density $\left(\rho=1 \mathrm{~kg} / \mathrm{m}^{3}\right)$. The imposed simple support conditions were: $w^{j}(x=0, y)=w^{j}(x=\alpha$, $y)=w^{j}(x, y=0)=w^{j}(x, y=\alpha)=0, v^{j}(x=0, y)=v^{j}(x=\alpha, y)=0, u^{j}(x, y=0)=u^{j}(x, y=\alpha)=0$. In addition, the outer surfaces of the piezoelectric layers were forced to remain always grounded. Based on this, two sets of electric boundary conditions were considered for the inner surfaces of the piezoceramic layers: a closed-circuit condition termed with (C), with the potential forced to remain zero (grounded); and an open-circuit condition termed with $(O)$, where the electric potential remains free (zero electric displacements).

Fundamental Natural Frequency. The predicted fundamental frequency $f_{1}$ of the plate with various uniform mesh densities and various numbers of discrete layers through the thickness of the laminate is shown in Table 2. In the case of three discrete-layers $(\mathrm{N}=4)$, one discrete layer was used for each piezoelectric ply and one for the whole composite sublaminate; in the case of 7 discrete layers $(N=8)$, 2 discrete layers were used for each piezoelectric ply and 1 for each composite ply; and in the case of 20 discrete layers $(\mathrm{N}=21), 4$ layers were used for each piezoelectric and composite ply. For both thickness ratios, the predicted natural frequencies consistently converge above and below the values of the exact solution depending on the type of electric boundary conditions, which shows that the element can model thick piezoelectric composite structures, while it does not lock at low thicknesses. Although the agreement is very good, the predicted range of the piezoelectric effect on the fundamental frequency (the difference between $(\mathrm{O})$ and $(\mathrm{C})$ conditions) is much higher in the FE results than in the exact solution, and the reason for this difference remains a subject of investigation. The consideration of minimal discrete-layers through the thickness seems to provide reasonable accuracy in the calculation of frequencies for both thickness ratios. Yet, higher number of discretelayers may be required in thick laminates to capture the local laminate response, as shown in subsequent examples. 
Electromechanical State. The through-the-thickness distributions of the modal displacements and the normalized electric potential of the fundamental mode of the plate at open-circuit conditions $(0)$ are shown in Figs. 2-3 for $a$ thin and thick plate, respectively. The transverse displacement $w$ and electric potential correspond to the center of the plate $(x=\alpha / 2, y=\alpha / 2)$, while the in-plane displacements $u$ and $v$ are at the midspan of the edges, e. g. $(x=\alpha, y=\alpha / 2)$ and $(x=\alpha / 2, y=\alpha)$, respectively. An 8 by 8 uniform mesh and 7 discrete layers through the thickness were used. In the case of the thin plate ( $\alpha / h=50$, Fig. 2) both w-constant and $w$-variable laminate theories and corresponding finite elements (FE) yielded identical results with the exact solution. In the case of the thick plate ( $\alpha / \mathrm{h}=4$, Fig. 3), both laminate theories (w-constant and w-variable) and corresponding finite elements yielded excellent agreement with the exact solution. The w-constant theory slightly underpredicted the in-plane displacements, and did not capture the slight variation in w (mostly due to the $d_{33}$ piezoelectric coefficient) in the piezoelectric layers. The variation of the electric potential in the composite plies was caused by the in-plane electric permittivity $\varepsilon_{11}$ and $\varepsilon_{22}$ of the composite plies. Clearly, the assumptions of simplified theories (simplified linear displacement and electric fields, single layer assumptions) are only valid for low thicknesses (Fig. 2), while significant deviations and errors may be observed in thick plates (Fig. 3).

Modal Stresses. The predicted modal stresses through the thickness of the laminate are shown in Figs 4-5, for the thin and thick plate, respectively. The stresses were calculated at the points of respective maximum values, that is, at the center $\left(\sigma_{x y} \sigma_{y}\right)$, the corner $\left(\sigma_{x y}\right)$, and the mid-edges of the plate $\left(\sigma_{y p} \sigma_{x y}\right)$. An $8 \times 8$ uniform finite element mesh was used with 20 discrete layers through the thickness. Calculated stresses with the w-constant finite element model are shown in Figs. 4-5, corresponding to the integration points and elements closer to the respective stress location. Again for both thickness ratios, the agreement of the finite element predictions to the exact solution is excellent. Fig. 5 shows clearly that in thick laminates, the stresses exhibit substantial nonlinearity and slope discontinuities between each ply, which can not be captured by simplified kinematic assumptions. The previous results and comparisons have demonstrated the excellent accuracy and robustness of the mechanics and the finite elements, and their capability to capture both the global 
and local through-the-thickness response of thick and thin piezoelectric plates.

Effect of Electric Conditions. Figs. 6a-d illustrate the effect in the modal in-plane displacements, electric potential, and stresses of the thick plate, when different conditions are imposed on the electric potential at the inner surfaces of the piezoelectric layers. Specifically, the cases of forced (equal to zero) and free electric potential are shown in Fig. 6, identified as closed-circuit (C) and open-circuit $(O)$ conditions respectively. As seen in Fig. 6a the voltage conditions have a definite effect on the modal in-plane displacements, as a result of the different electric fields in the piezoelectric layers (see Fig. 6b). It is interesting to note that electric fields exist in the piezoelectric layers even with the closed-circuit conditions. The effects of electric conditions are more profound in the in-plane stress $\sigma_{x}$ (Fig. 6c) and the interlaminar shear stresses (Fig. 6d), and are attributed primarily to the differences in the piezoelectric stress component (see eq. (2)).

\section{Composite Plates with Discrete Piezoelectric Patches}

The present applications illustrate the natural capability of the finite element to model more realistic configurations of smart structures, that is: (a) discrete piezoelectric actuators/sensors, and (b) continuous electrodes on the free surfaces which force the sensory electric potential to remain uniform. The plates considered in this example and the corresponding meshes are shown in Fig. 7. The first is a [0/ 45 ], Gr/Epoxy cantilever plate with 15 piezoceramic patches at each side built and tested by Crawley and Lazarus (1989); the second is a candidate [ $\left.\mathrm{p}_{2} / 0 / 90 / 45 /-45\right], \mathrm{T} 300 / 934$ Gr/Epoxy cantilever plate with 8 square PZT-4 patches at each side with properties shown in Table 1. Both plates were modeled using the w-constant theory with 3 discrete layers.

[0/土45], Cantilever Plate. Fig. 8 shows the predicted transverse deflection of the $0.83 \mathrm{~mm}$ thick [0/145], Gr/Epoxy (AS4/3501) plate shown in Fig. 7a with 15 piezoceramic patches bonded on each side, each $0.25 \mathrm{~mm}$ thick. The deflections are induced by an applied uniform electric field of 394 $\mathrm{V} / \mathrm{mm}$, of opposite polarity at the upper and lower piezoelectric patches. Measured data were reported by Crawley and Lazarus (1989), together with some material properties (additional material 
properties were backcalculated as required from related data in the same publication). The following normalized form was used for the deflections consistent with the way the experimental results were published,

$$
T_{1} \cdot w_{2} / c, \quad T_{2}=\left(w_{2}-\left(w_{1}+w_{3}\right) / 2\right) / c, \quad T_{3}-\left(w_{3}-w_{1}\right) / c
$$

where, $w_{2}, w_{1}, w_{3}$ are the transverse displacement along the midline and the two edges respectively, and $c$ is the width of the plate. The three normalized displacements respectively represent or approximate the axial bending deflection, the transverse bending curvature, and the twisting angle due to bending-twisting coupling. There is very good agreement between predicted and measured transverse deflection $T_{1}$. The agreement between predicted and measured transverse curvature $T_{2}$ and twisting angle $T_{3}$ is fair to good. The present predictions and correlation trends agree remarkably well with similar results reported by $\mathrm{Ha}$ et al. (1992), hence, the differences were attributed to uncertainties in the material properties and possible specimen imperfections.

[p/ /0/90/45/-45], Cantilever Plate. Figs. 9-10 show the predicted first two electromechanical modes of this plate (Fig. 7b). All piezoceramic layers were configured to act as sensors, with continuous electrodes on each surface, which were represented with equality constraints imposed on the respective electric potential. There is a characteristic modal voltage distribution corresponding to the characteristic distortion of each mode. The first mode (Fig. 9) is almost a pure first bending mode in the axial direction. The second mode (Fig. 10) is the first torsional mode in the axial direction. Both figures indicate the non-apparent relationship between deflected shape and the corresponding sensory voltage pattern. The slight asymmetries in the sensory voltage patterns are caused by the flexuretwisting coupling of the laminate. Figs. 9-10 clearly illustrate the various static and/or dynamic deformations which may be actively induced, for example bending or twisting, by applying the proper pattern of voltage on the piezoelectric elements. Note also that undesirable deformations, caused by laminate coupling, may be annihilated in this manner. The natural calculation of the characteristic voltage distributions is an obvious advantage of the method, because it provides the voltage patterns required to induce the corresponding deflection, or to construct the modal filter which can be used to isolate, sense and ultimately control the respective mode. 
Overall, all previous application cases have demonstrated the accuracy, quality and versatility of the developed laminate theory and corresponding finite elements, and their capability to model a broad array of global and local responses in most practical configurations of active/sensory piezoelectric composite plates.

\section{Summary}

Laminate and structural mechanics and the corresponding coupled electromechanical models for the dynamic analysis of smart composite plate structures with embedded piezoelectric actuators and sensors were developed and described. The described laminate theories entail mixed displacement and electric field formulations and implement layerwise representations of the displacements and electric potential. A robust linear plate finite element, in terms of thickness, was also formulated. The mechanics have capabilities to simulate both sensory and active dynamic response of smart composite structures either at the global structural or the local laminate level.

Correlations of predicted results with exact solutions of simply-supported piezoelectric composite plates have demonstrated the accuracy, robustness and versatility of the developed mechanics. The comparisons verified the capability of the present mechanics to model the free-vibration of thick and/or thin piezoelectric composite plates. Additional application studies have demonstrated the capability of the mechanics to model the active and sensory, static and dynamic response of smart composite plates with continuous or discrete piezoceramic devices. Finally, the applications have demonstrated the possibilities for dynamic displacement management provided by smart piezoelectric composite structures.

\section{Acknowledgement}

This work was supported by NASA Cooperative Agreement NCC3-391. This support is gratefully acknowledged. 


\section{References}

Allik H. and Hughes T. J. R. (1970). Finite Element for Piezoelectric Vibration. International J. for Numerical Methods in Engineering 2, 151-157.

Crawley E. F. and Lazarus K. B. (1989). Induced Strain Actuation of Composite Plates. ALAA Paper 89-1326-CP, 30th AIAA Structural Dynamics and Materials Conference, Mobile, Alabama.

Ha S. K., Keilers C. and Chang F. K. (1992). Finite Element Analysis of Composite Structures Containing Distributed Piezoceramic Sensors and Actuators. AIAA J. 30:3, 772-780.

Heyliger P. R, Ramirez G. and Saravanos D. A. (1994). Coupled Discrete-Layer Finite Elements for Laminated Piezoelectric Plates. Communications in Numerical Methods in Engineering 10, 971-981.

Heyliger P. R and Saravanos D. A. (1995). Exact Free-Vibration Analysis of Laminated Plates with Embedded Piezoelectric Layers. J. of Acoustical Society of America 98:3, 1547-1557.

Lammering R. (1991). The Application of a Finite Shell Element for Composites Containing Piezoelectric Polymers in Vibration Control. Computers and Structures 41, 1101-1109.

Lee C. K. and Moon F. C. (1989). "Laminated Piezopolymer Plates for Torsion and Bending Sensors and Actuators. J. of the Acoustical Society of America 85, 2432-2439.

Lee C. K. (1990). Theory of Laminated Piezoelectric Plates for the Design of Distributed Sensors/Actuators. Part I: Governing Equations and Reciprocal Relationships. J. of the Acoustical Society of America 87, 1144-1158.

Lee C. K. and Moon F. C. (1990). Modal Sensors/Actuators. J. of Applied Mechanics 57, 434-441. 
Naillon M., Coursant R. H. and Besnier F. (1983). Analysis of Piezoelectric Structures by a Finite Element Method. Acta Electronica 25, 341-362.

Pauley K. P. (1974). Analysis of Plane Waves in Infinite, Laminated, Piezoelectric Plates, Ph.D. Dissertation, University of California at Los Angeles.

Reddy J. N. (1987) A Generalization of Displacement-Based Laminate Theories. Communications in Applied Numerical Methods 3, 173-181.

Robbins D. H. and Reddy J. N. (1991). Analysis of Piezoelectrically Actuated Beams Using a Layer-Wise Displacement Theory. Computers and Structures 41, 265-279.

Robbins D. H. and Reddy J. N. (1993). Modelling of Thick Composites Using a Layerwise Laminate Theory. International J. for Numerical Methods in Engineering 36, 655-677.

Saravanos D. A. (1993). Analysis of Passive Damping in Thick Composite Structures. AIAA J. 31:8, 1503-1510.

Saravanos D. A (1994). Integrated Damping Mechanics for Thick Composite Laminates and Plates. J. of Applied Mechanics 61:2, 375-383.

Saravanos D.A and Heyliger P. R. (1995). Coupled Layerwise Analysis of Composite Beams with Embedded Piezoelectric Sensors and Actuators. J. of Intelligent Material Systems and Structures 6:3, pp. $350-363$.

Tiersten H. F. (1969). Linear Piezoelectric Plate Vibrations, Plenum Press, New York.

Tzou H. S. and Tseng C. I. (1990). Distributed Piezoelectric Sensor/Actuator Design for Dynamic Measurement/Control of Distributed Parametric Systems: A Piezoelectric Finite Element Approach. 
J. of Sound and Vibration 138, 17-34.

Wang B. T. and Rogers C. A (1991). Laminate Plate Theory for Spatially Distributed Induced Strain Actuators. J. of Composite Materials 25, 433-452. 
Table 1 Mechanical Properties

$\left(\varepsilon_{0}=8.8510^{-12} \mathrm{farad} / \mathrm{m}\right.$, electric permittivity of air)

\begin{tabular}{|c|c|c|}
\hline Property & Gr/Epoxy & PZT-4 \\
\hline \multicolumn{3}{|l|}{ Elastic Properties: } \\
\hline $\mathrm{E}_{11} \quad(\mathrm{GPa})$ & 132.38 & 81.3 \\
\hline $\mathrm{E}_{22} \quad(\mathrm{GPa})$ & 10.76 & 81.3 \\
\hline $\mathrm{E}_{33} \quad(\mathrm{GPa})$ & 10.76 & 64.5 \\
\hline $\mathrm{G}_{23} \quad(\mathrm{GPa})$ & 3.61 & 25.6 \\
\hline $\mathrm{G}_{13} \quad(\mathrm{GPa})$ & 5.65 & 25.6 \\
\hline $\mathrm{G}_{12} \quad(\mathrm{GPa})$ & 5.65 & 30.6 \\
\hline$v_{12}$ & 0.24 & 0.33 \\
\hline$v_{13}$ & 0.24 & 0.43 \\
\hline$v_{23}$ & 0.49 & 0.43 \\
\hline \multicolumn{3}{|c|}{ Piezoelectric coefficients $\left(10^{-12} \mathrm{~m} / \mathrm{V}\right)$} \\
\hline$d_{31}$ & 0 & -122 \\
\hline$d_{32}$ & 0 & -122. \\
\hline$d_{33}$ & 0 & 285. \\
\hline$d_{24}$ & 0 & 0. \\
\hline \multicolumn{3}{|l|}{ Electric Permittivity: } \\
\hline$\varepsilon_{11} / \varepsilon_{\mathrm{o}}$ & 3.5 & 1475. \\
\hline$\varepsilon_{22} / \varepsilon_{0}$ & 3.0 & 1475. \\
\hline$\varepsilon_{33} / \varepsilon_{0}$ & 3.0 & 1300. \\
\hline Mass Density $\rho\left(k g / \mathrm{m}^{3}\right)$ & 1578. & 7600. \\
\hline
\end{tabular}


Table 2. Predicted Natural Frequencies of $[\mathrm{p} / 0 / 90 / 0 / \mathrm{p}]$ square simply supported Plate. (C)- closed circuit; (O)- open circuit

\begin{tabular}{|c|c|c|c|c|c|}
\hline \multirow[t]{2}{*}{ Mesh Density } & \multirow[t]{2}{*}{$\begin{array}{l}\text { Discrete Layers } \\
(N-1)\end{array}$} & \multicolumn{4}{|c|}{$\begin{array}{l}\text { Fundamental Frequency, } f_{l} a^{2} / \mathrm{h} \rho^{1 / 2} \\
\qquad 10^{3} \mathrm{~Hz}(\mathrm{~kg} / \mathrm{m})^{1 / 2}\end{array}$} \\
\hline & & $\alpha h=4$ (C) & $\alpha h=4(O)$ & $\alpha h=50$ (C) & $\alpha / h=50$ (O) \\
\hline \multicolumn{6}{|l|}{$w$-constant } \\
\hline $4 \times 4$ & 3 & 148.154 & 153.720 & 249.860 & 272.860 \\
\hline $8 \times 8$ & 3 & 145.323 & 151.222 & 236.833 & 259.173 \\
\hline $12 \times 12$ & 3 & 144.768 & 150.727 & 234.533 & 256.765 \\
\hline $8 \times 8$ & 7 & 142.469 & 148.351 & 236.785 & 259.103 \\
\hline $8 \times 8$ & 20 & 142.221 & 148.177 & 236.784 & 259.102 \\
\hline \multicolumn{6}{|l|}{ w-variable: } \\
\hline $4 \times 4$ & 3 & 149.310 & 154.591 & 253.670 & 276.185 \\
\hline $8 \times 8$ & 3 & 146.269 & 151.964 & 239.628 & 261.703 \\
\hline $12 \times 12$ & 3 & 145.675 & 151.531 & 237.158 & 259.655 \\
\hline $8 \times 8$ & 7 & 142.942 & 148.692 & 237.383 & 259.630 \\
\hline $8 \times 8$ & 20 & 142.630 & 148.586 & 237.109 & 259.895 \\
\hline Exact: & & 145.339 & 145.377 & 245.941 & 245.942 \\
\hline
\end{tabular}




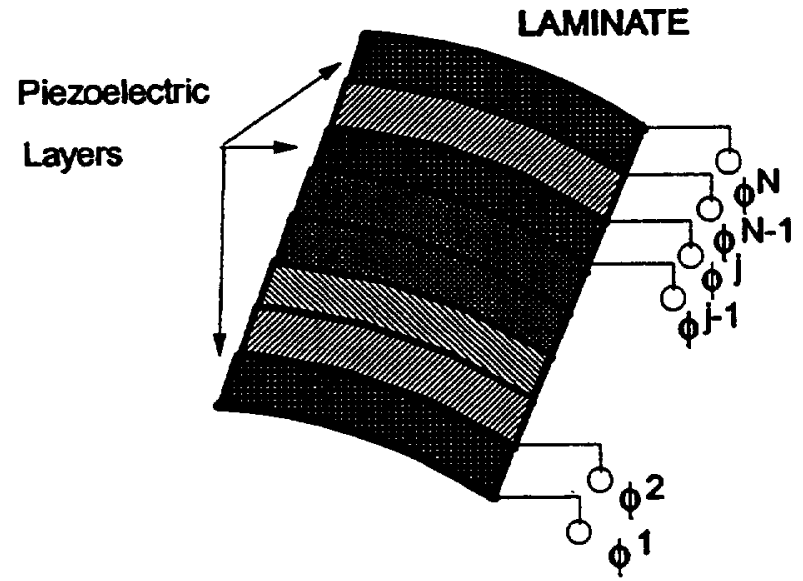

(a) Smart Laminate

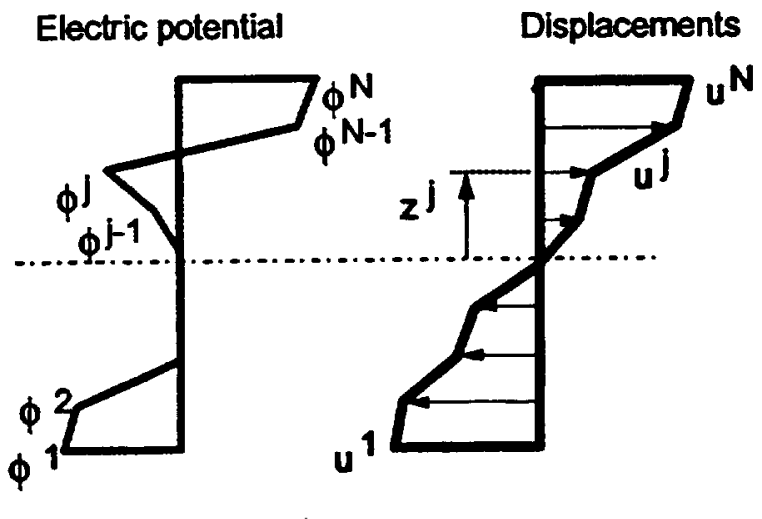

(b) Discrete-layer laminate theory assumptions

Figure 1.- Typical piezoelectric laminate configuration . (a) Concept ; (b) Assumed through-thethickness displacement and electric potential fields. 

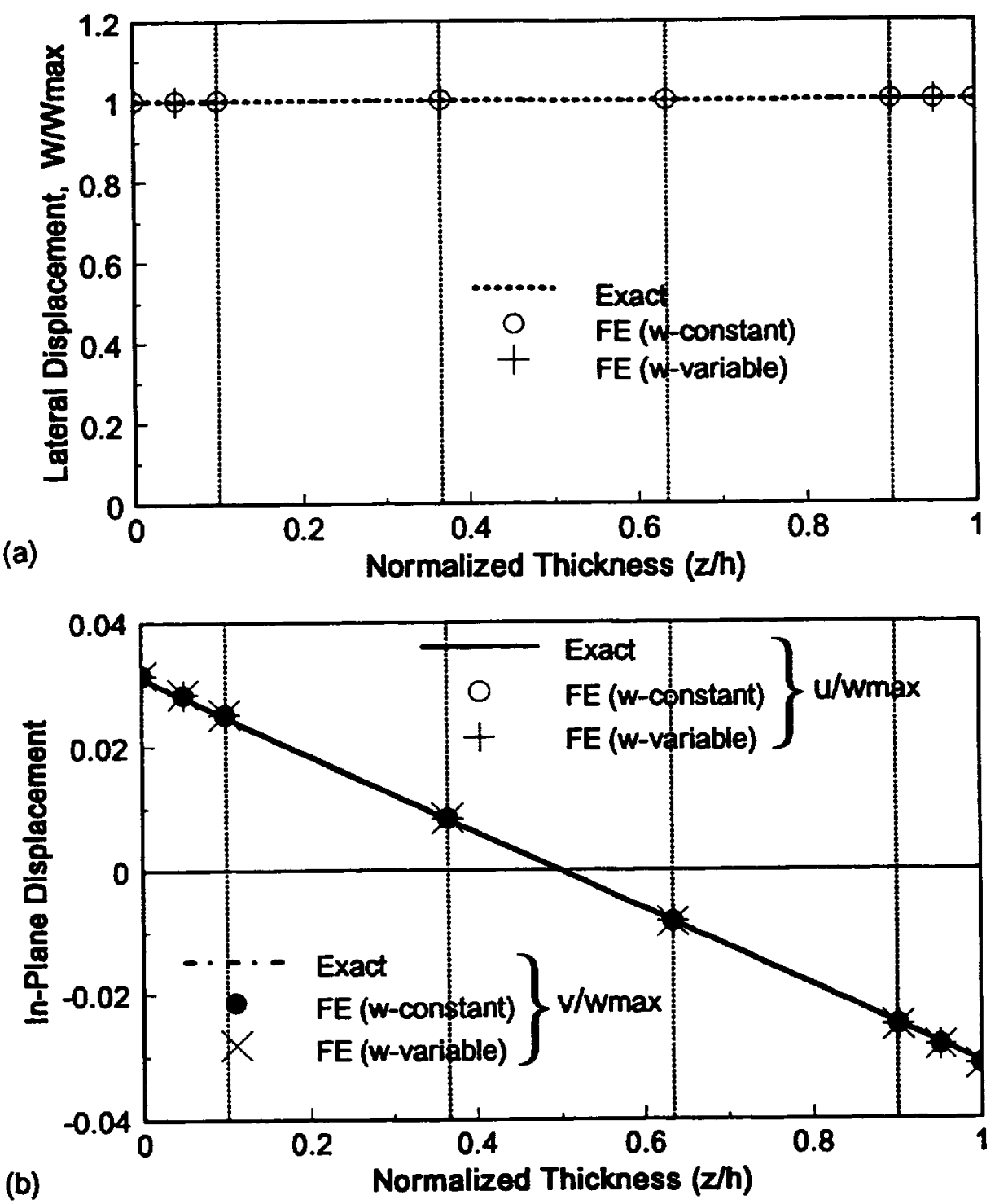

(b)

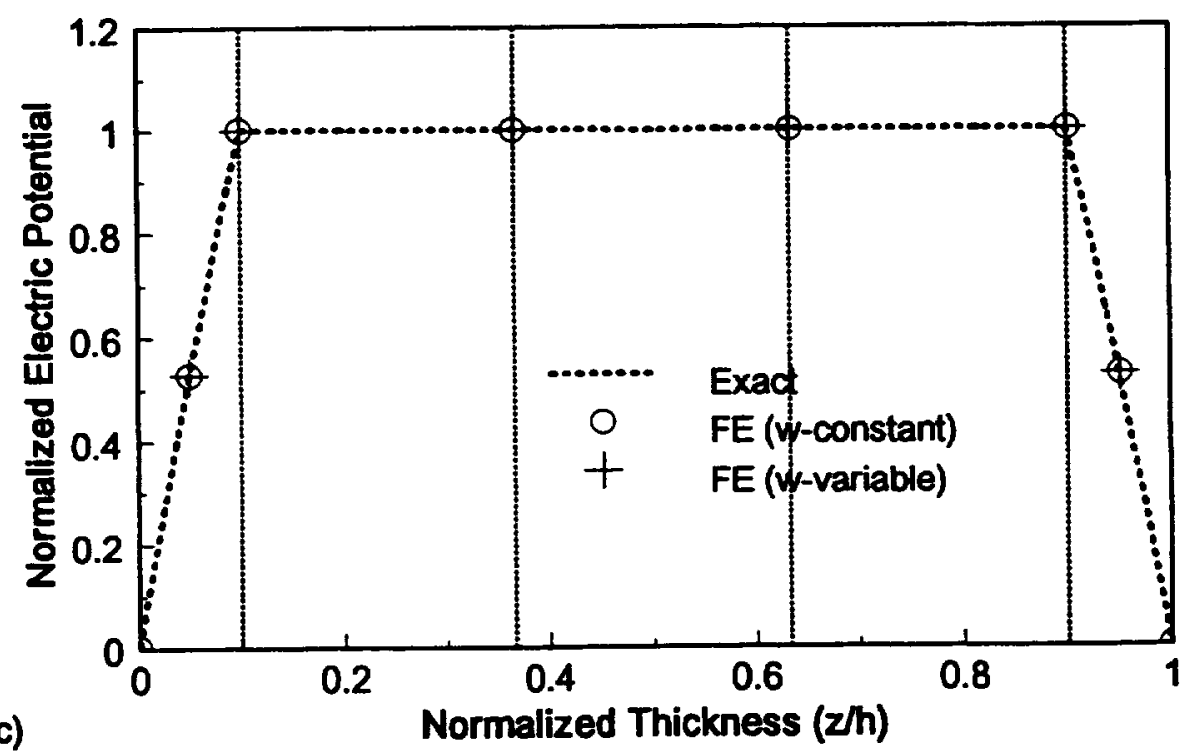

Figure 2. - Fundamental mode in a thin [p/0/90/0/p] simply supported square plate (thickness aspect ratio $\alpha / h=50$ ). (a) Lateral displacement $w / w_{\max }$; (b) in-plane displacements $w / w_{\max }$ and $v / w_{\operatorname{mxx}} ;(c)$ normalized electric potential $\phi / \phi_{\max }$. 
(a)

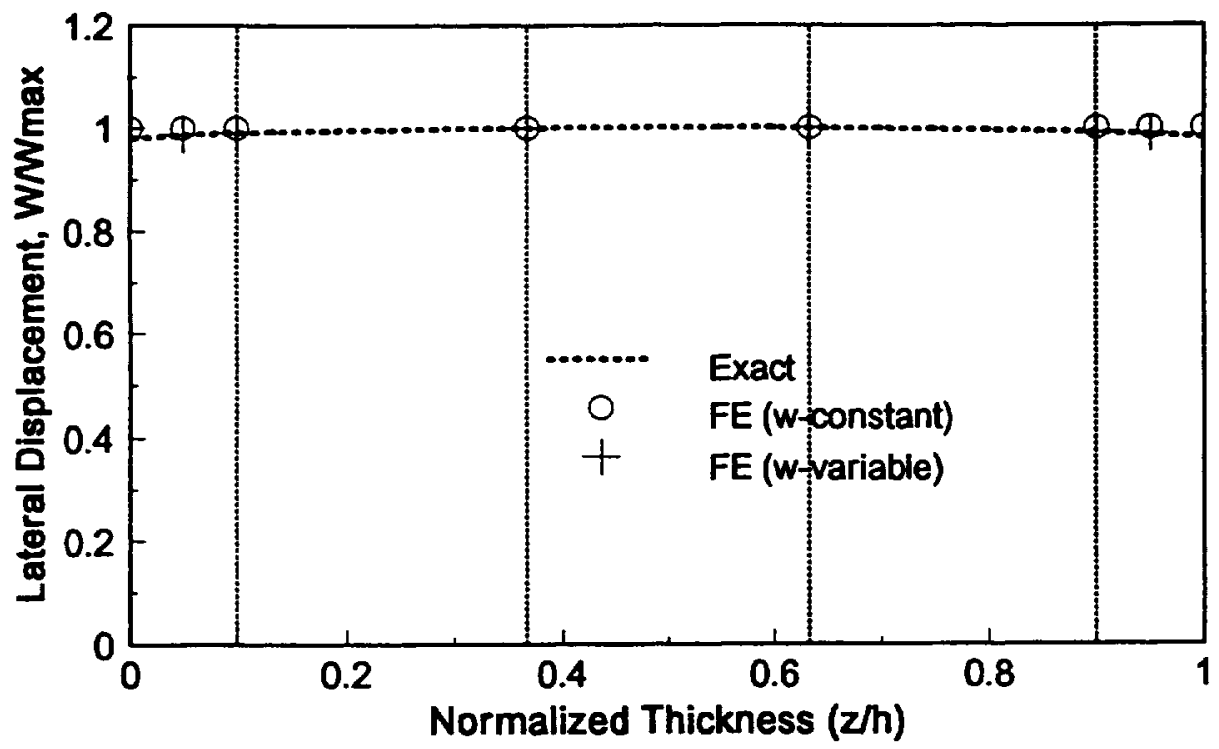

(b)

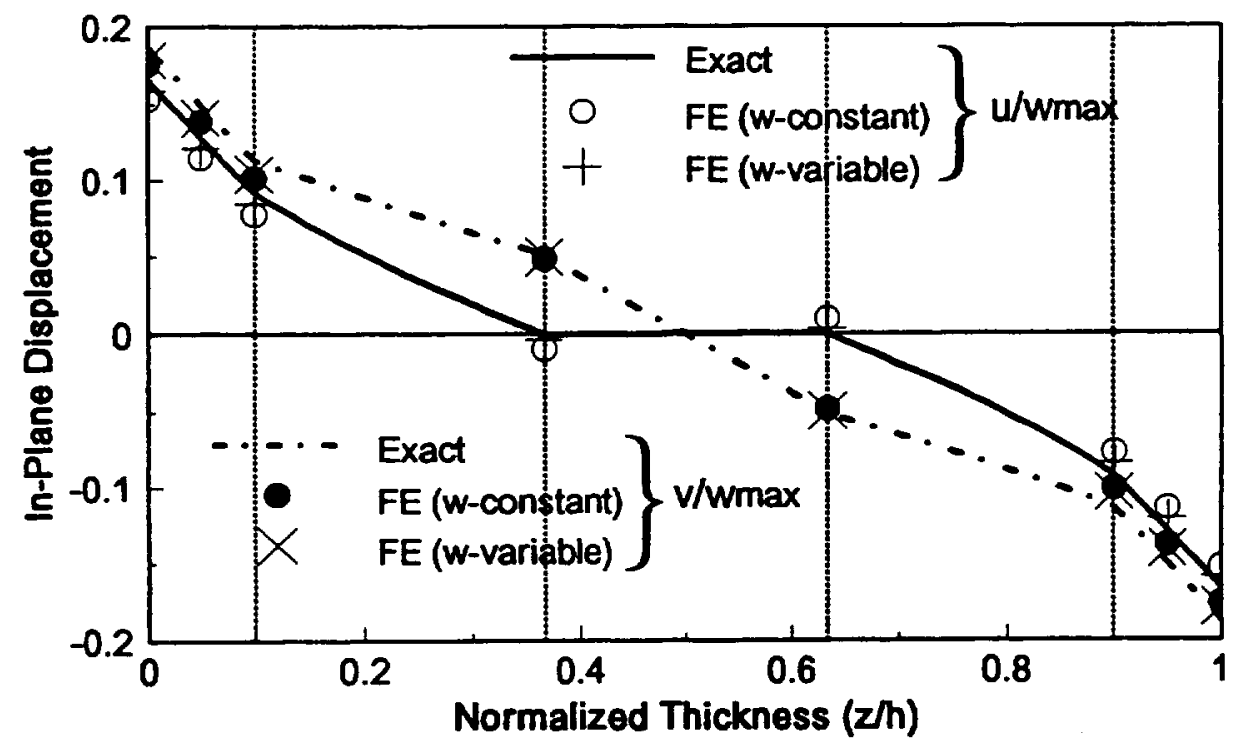

(c)

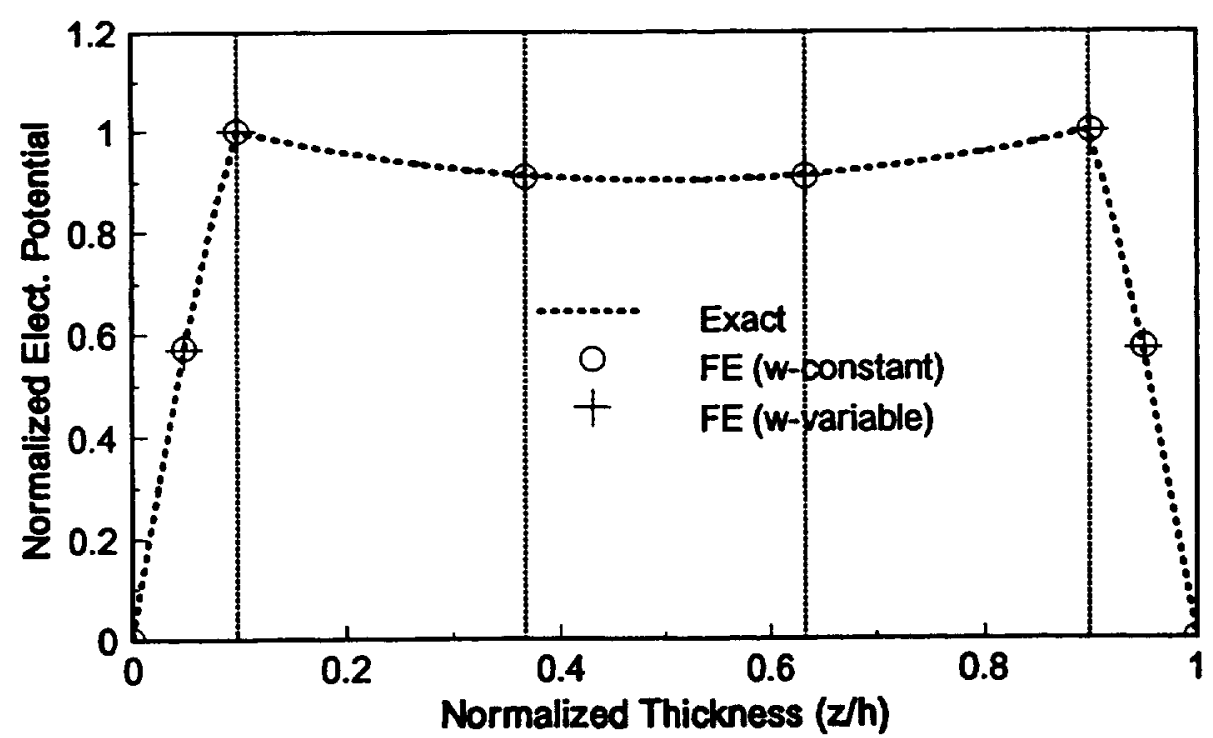

Figure 3.- Fundamental mode in a thick [p/0/90/0/p] simply supported square plate (thickness aspect ratio $\alpha / h=4$ ). (a) Lateral displacement $w / w_{\max }$ (b) in-plane displacements $u / w_{\max }$ and $\mathrm{v} / \mathrm{w}_{\max }$; (c) normalized electric potential $\phi / \phi_{\max }$. 

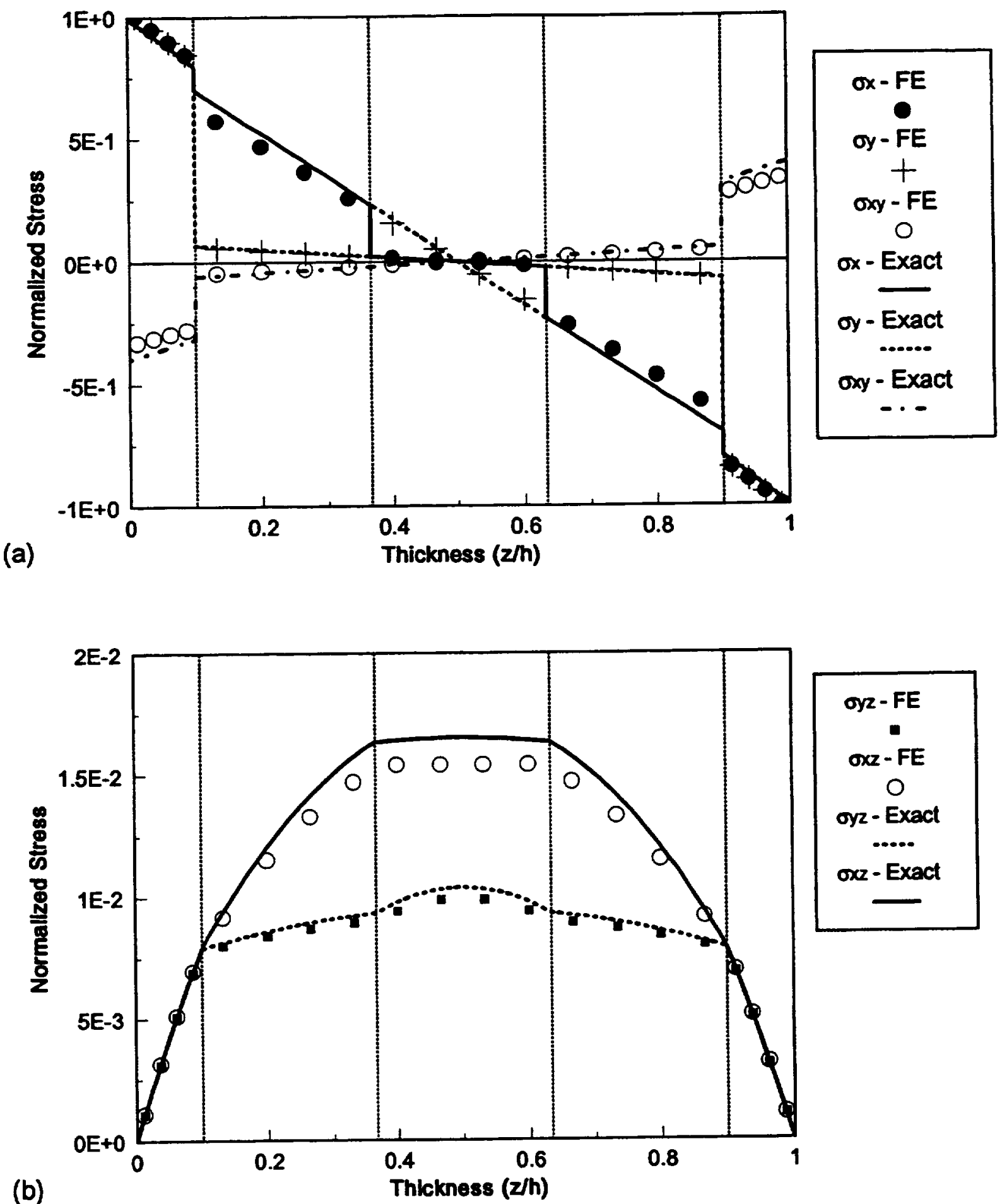

Figure 4. - Modal stress fields of the fundamental mode in a thin [p/0/90/0/p] simply supported square plate $(\alpha / \mathrm{h}=50)$. (a) In-plane stresses $\sigma_{x} /\left(\sigma_{y}\right)_{\max }, \sigma_{y} /\left(\sigma_{y}\right)_{\max }, \sigma_{x y} /\left(\sigma_{y}\right)_{\max } ;$ (b) interlaminar stresses $\sigma_{y z} /\left(\sigma_{y}\right)_{\operatorname{mex}} \sigma_{x z} /\left(\sigma_{y}\right)_{\max }$. 

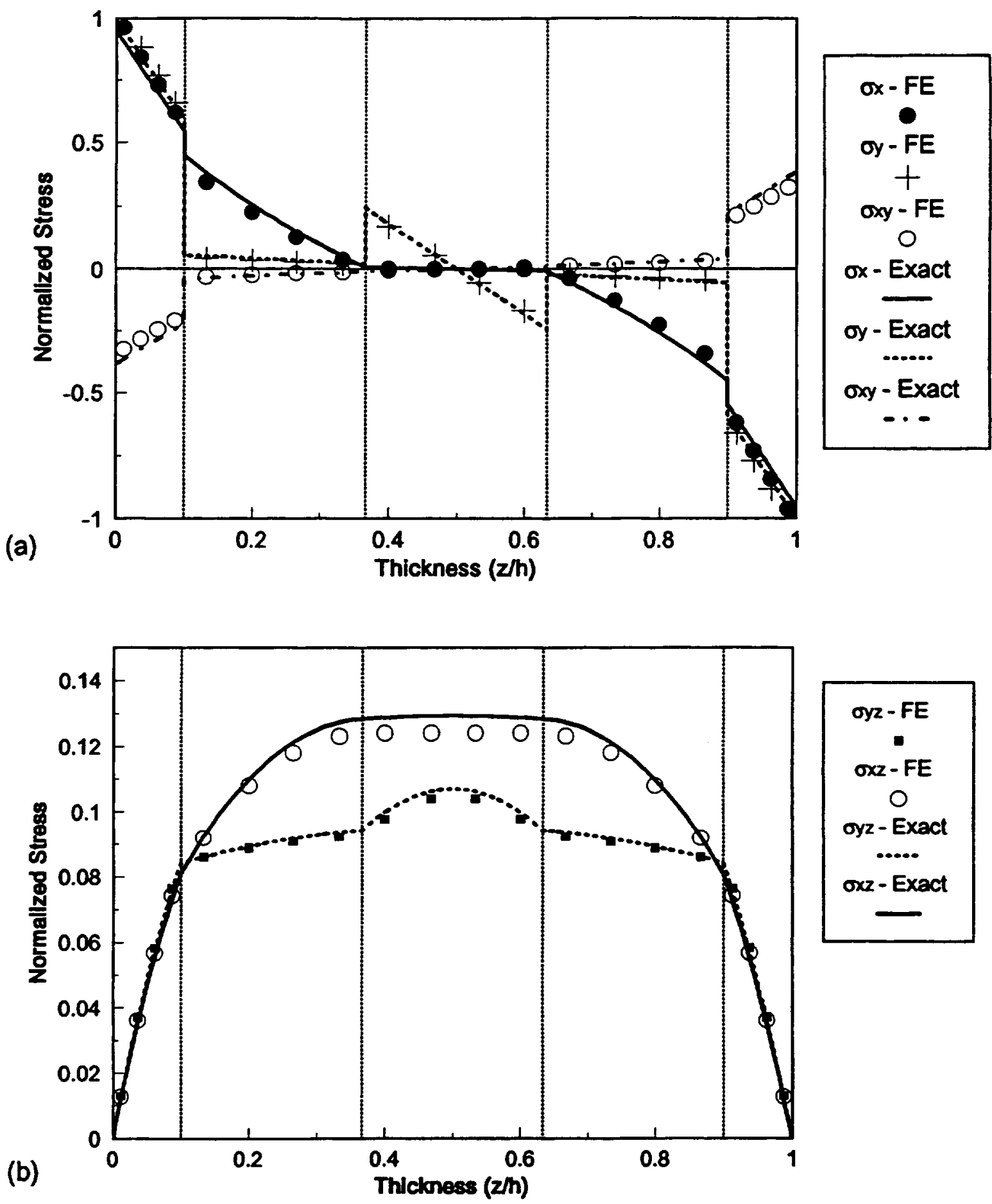

Figure 5.- Modal stress fields of the fundamental mode in a thick [p/0/90/0/p] simply supported square plate $(\alpha / \mathrm{h}=4)$. (a) In-plane stresses $\sigma_{x} /\left(\sigma_{y}\right)_{\max }, \sigma_{y} /\left(\sigma_{y}\right)_{\max }, \sigma_{x y} /\left(\sigma_{y}\right)_{\max }$ (b) interlaminar stresses $\sigma_{y z} /\left(\sigma_{y}\right)_{\max } \sigma_{z z} /\left(\sigma_{y}\right)_{\max }$. 

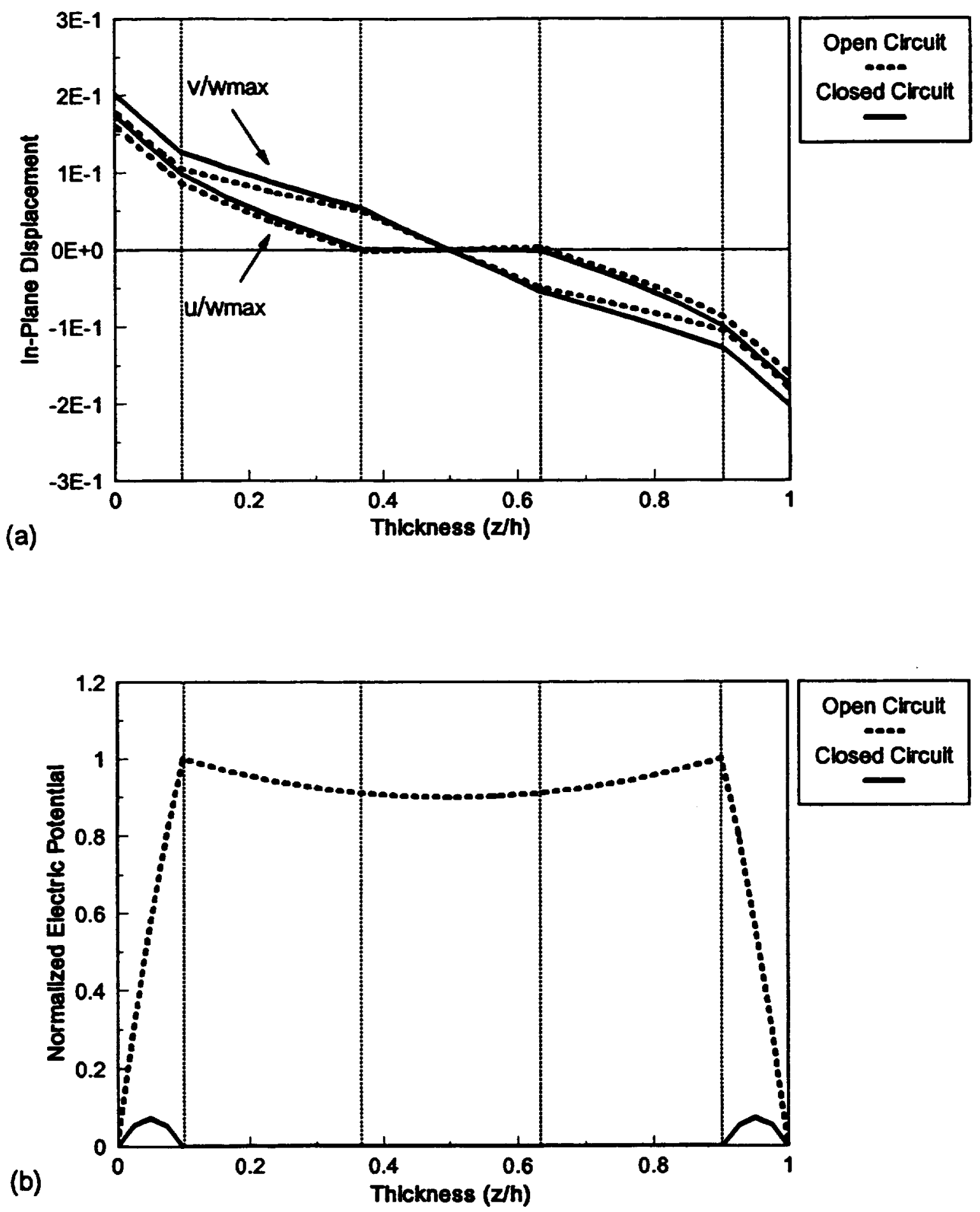

Figure 6.- Effect of electric boundary conditions on the fundamental mode of the thick [p/0/90/0/p] plate $(\alpha / h=4)$. (a) in-plane displacements $w / w_{\max }$ and $v / w_{\max }$; (b) normalized electric potential $\phi / \phi_{\max } ;(c)$ in-plane stress $\sigma_{x} /\left(\sigma_{y}\right)_{\max }(d)$ interlaminar stresses $\sigma_{y z} /\left(\sigma_{y}\right)_{\max } \sigma_{x z}$ $/\left(\sigma_{y}\right)_{\max }$. 


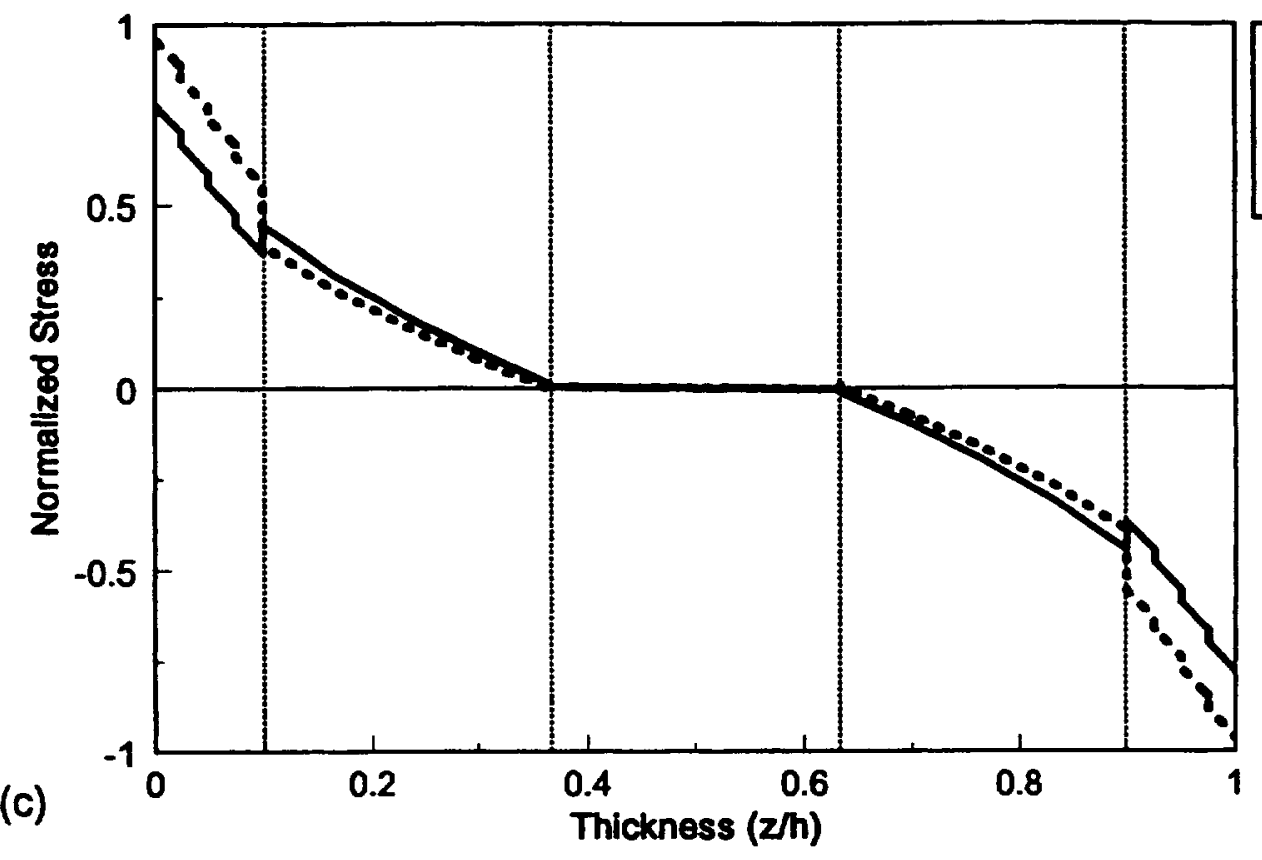

Open Circuit

Closed Circuit -

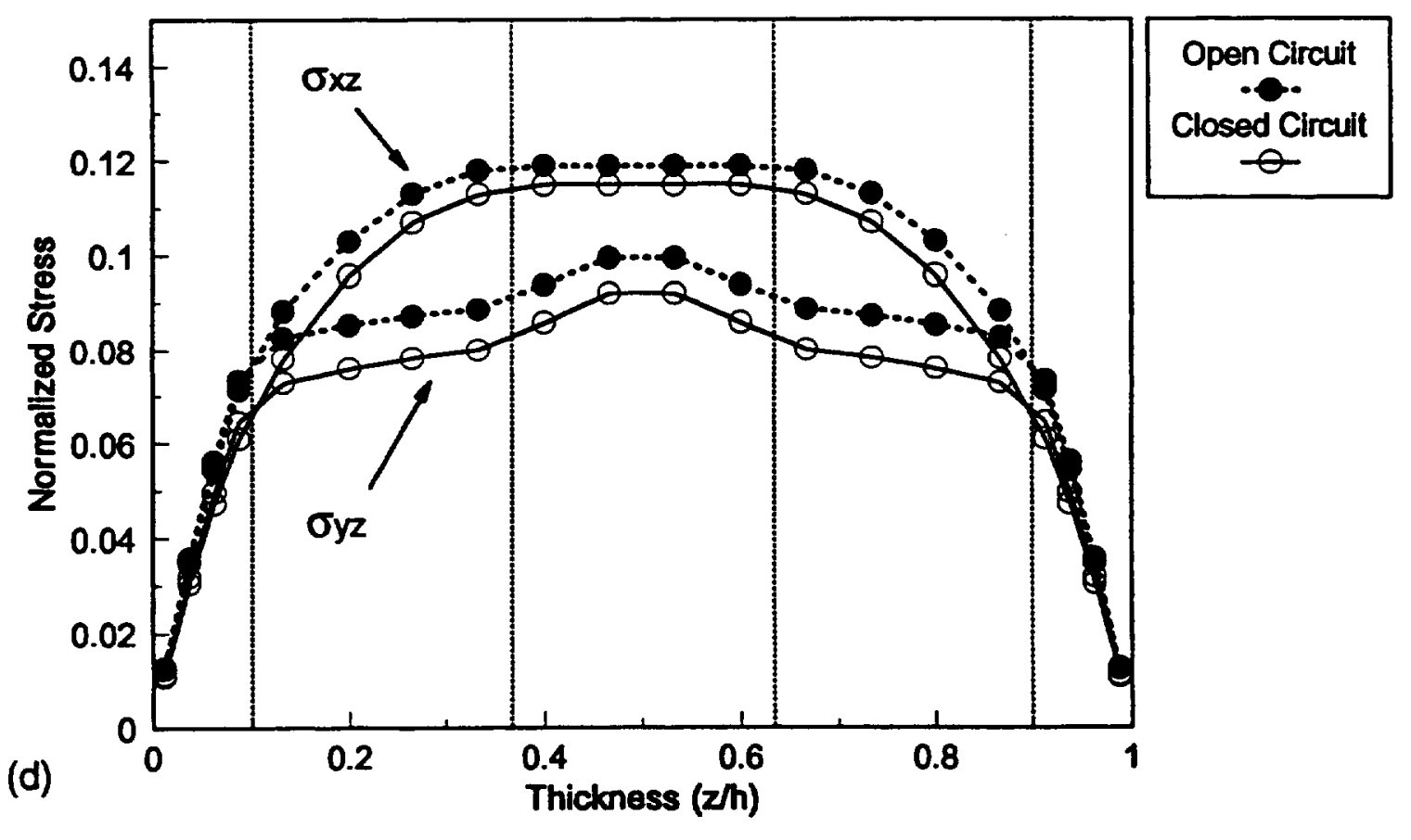

Figure 6.-(Continued) 

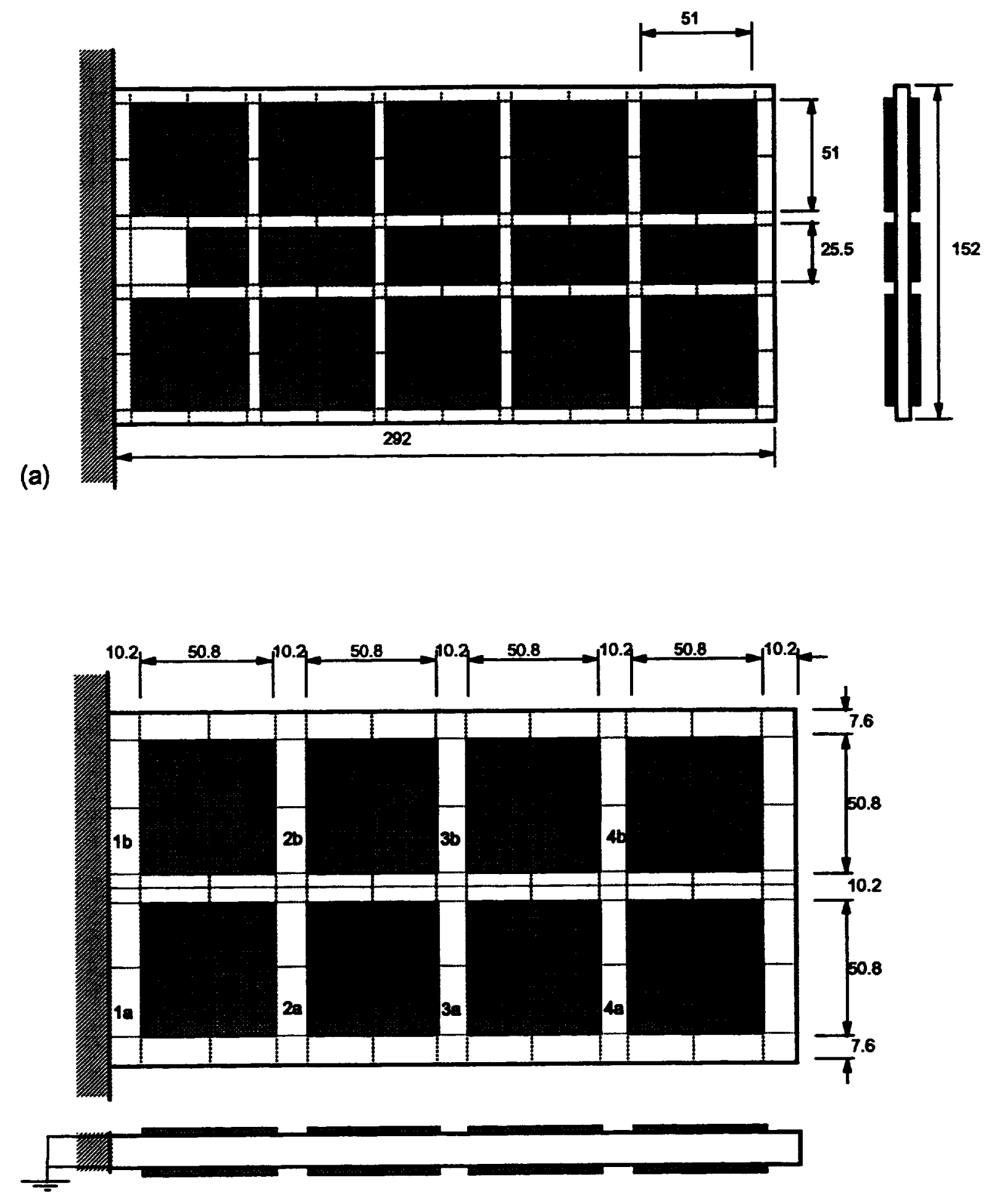

(b)

Figure 7.- Schematic configuration of cantilever plate beams with distributed piezoelectric patches.

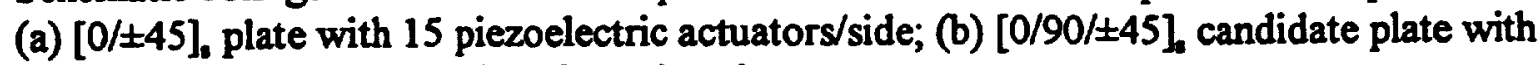
8 piezoelectric actuators/side. Dimensions in $\mathrm{mm}$. 

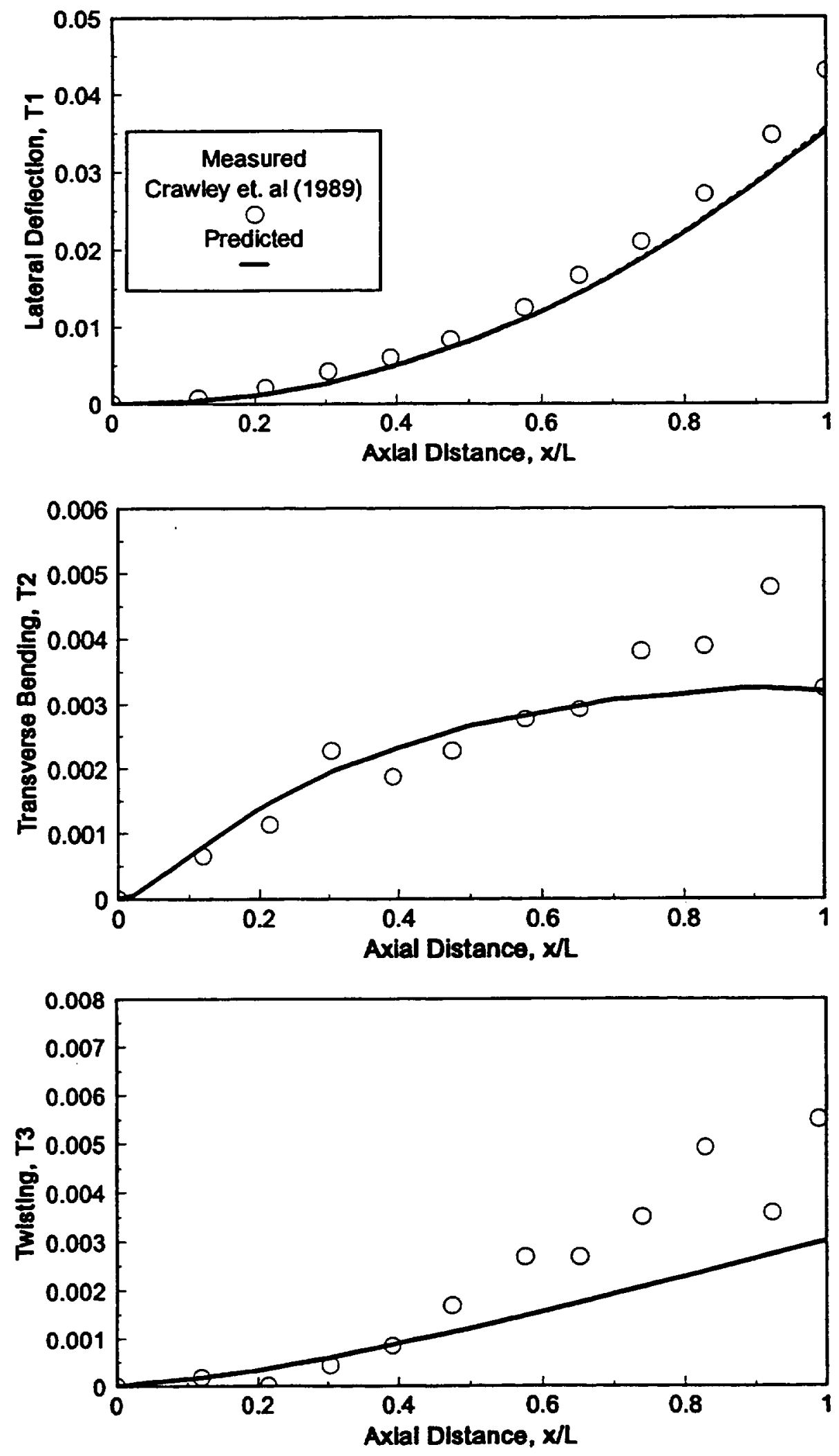

Figure 8. - Predicted induced deformations of the $[0 / \pm 45]_{3}$ cantilever plate beam. Measurements by Crawley and Lazarus (1989). 


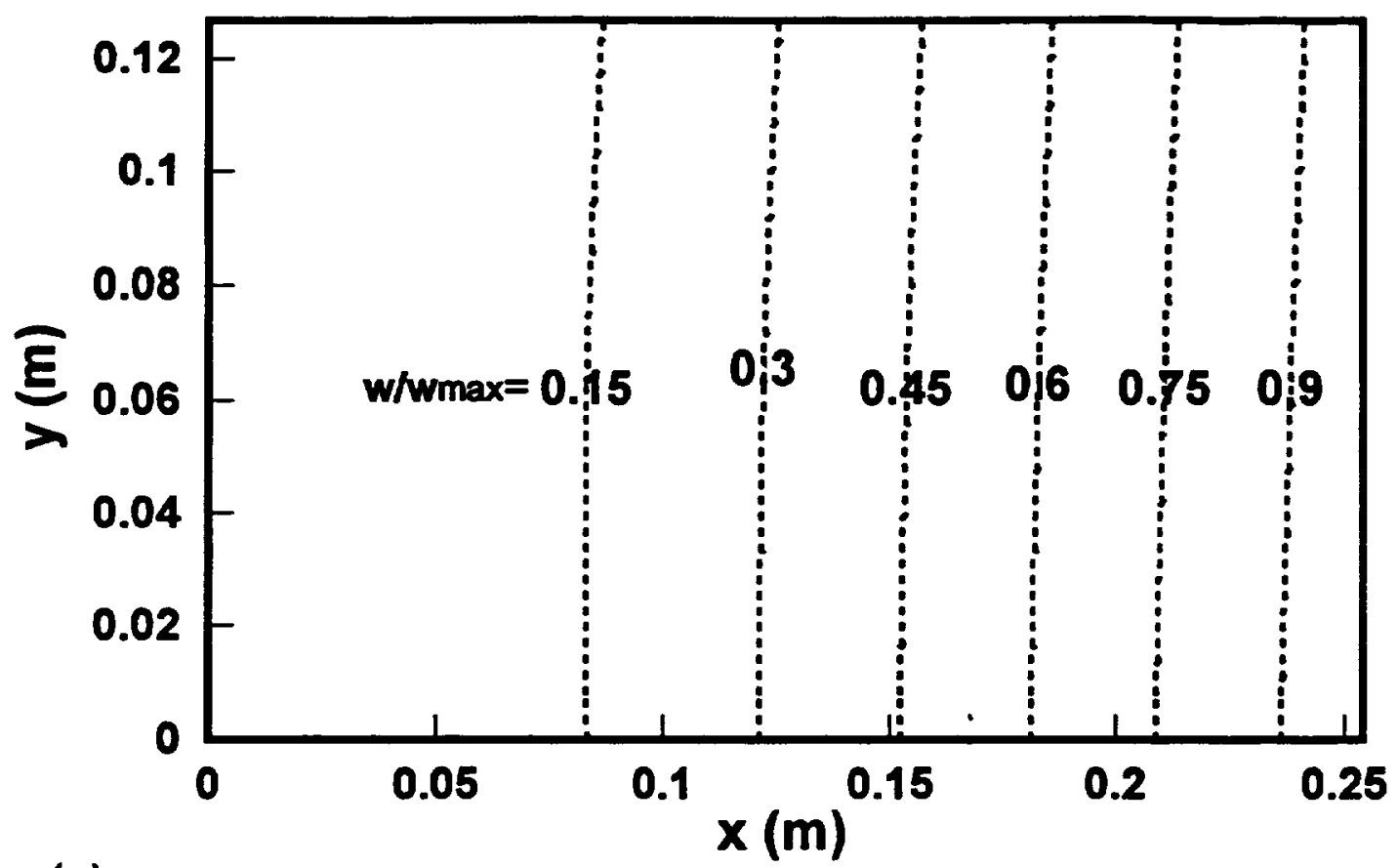

(a)

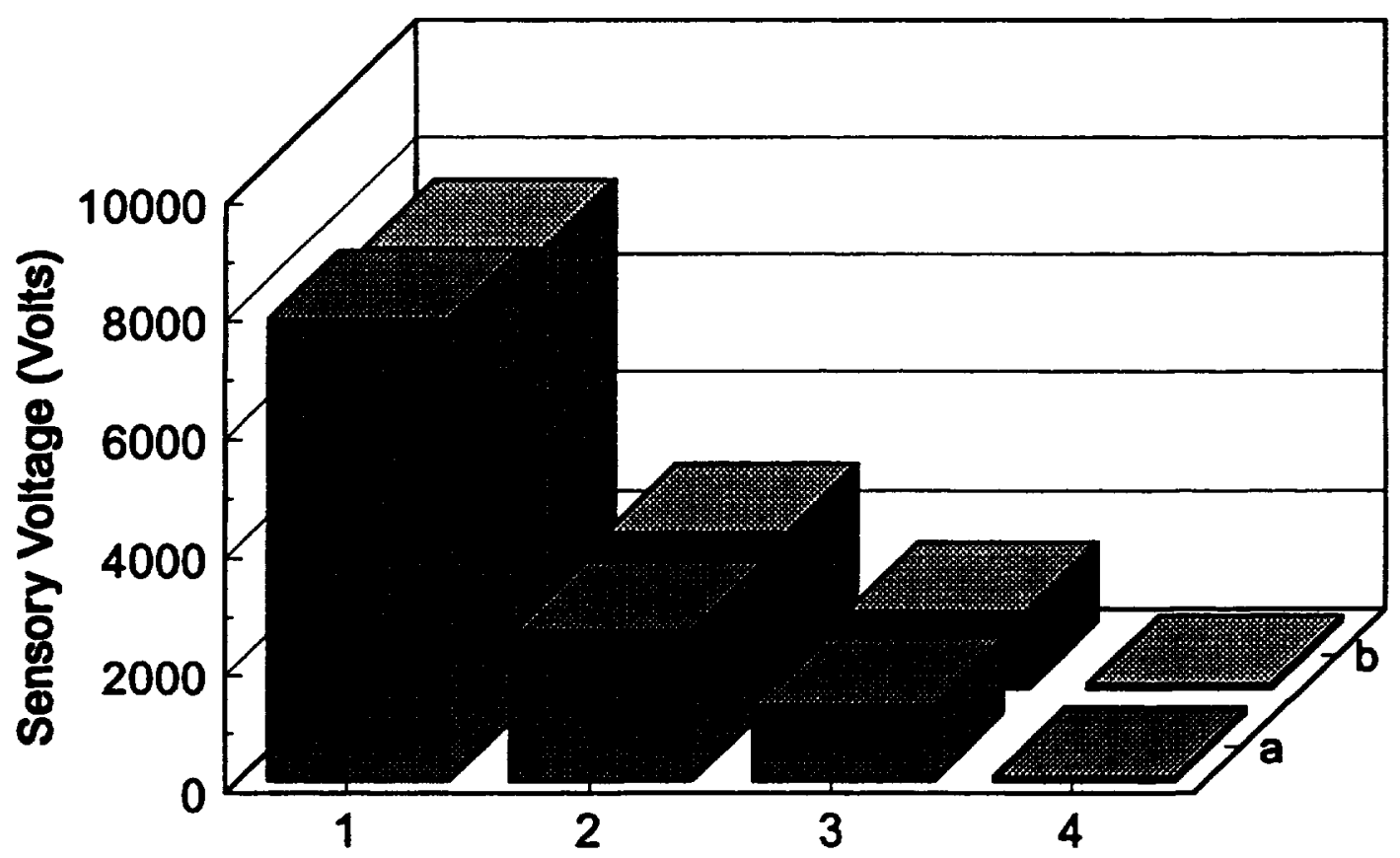

(b)

Figure 9. - First electromechanical mode of the $[0 / 90 / \pm 45]_{2}$ cantilever plate with discrete PZT -4 patches (1st bending). (a) Lateral deflections $w / w_{\max } ;$ (b) modal sensory voltage (upper surface). 

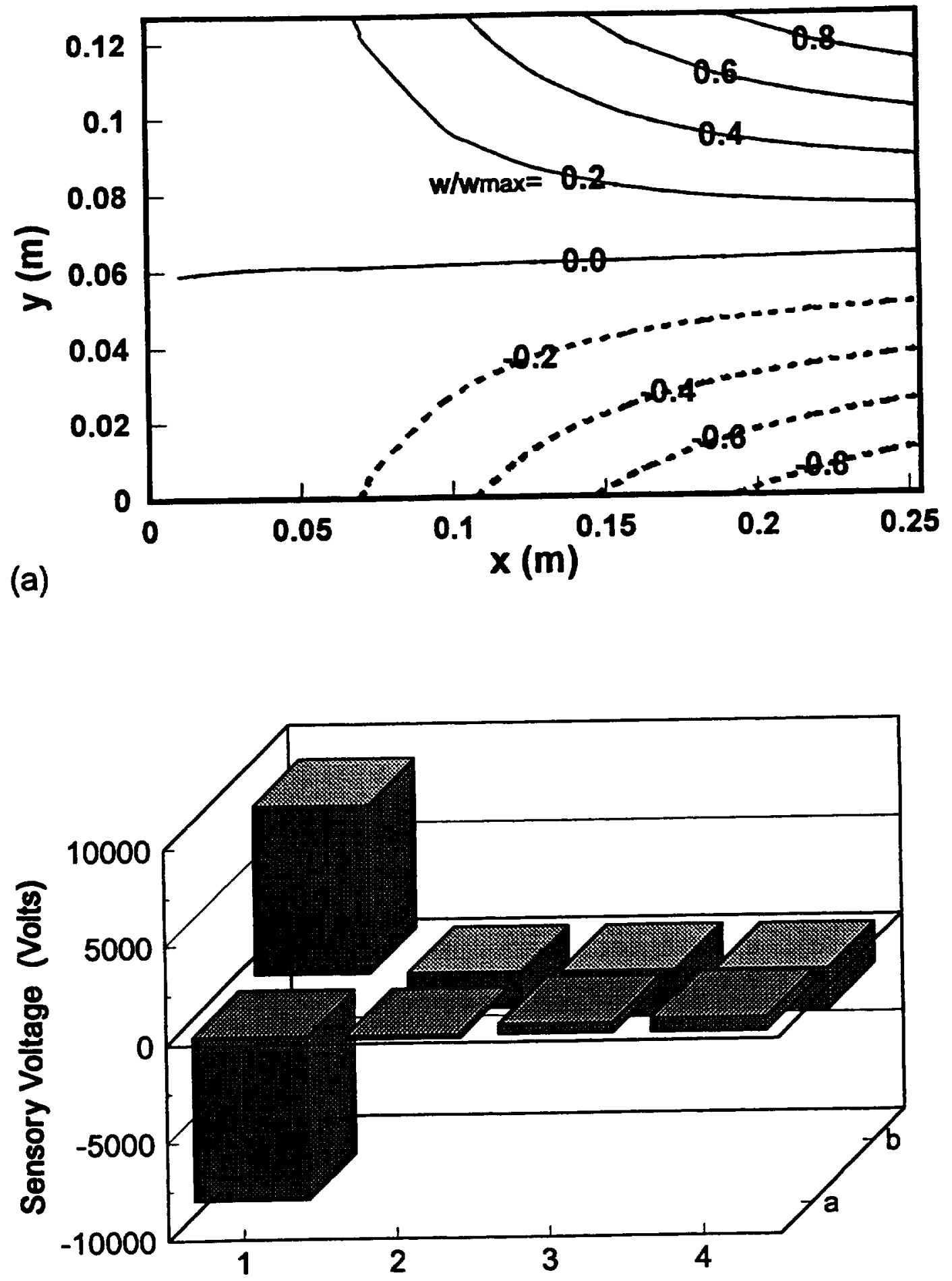

(b)

Figure 10.-Second electromechanical mode of $[0 / 90 / \pm 45]_{3}$ cantilever plate with discrete PZT-4 patches (1st torsion). (a) Lateral deflections $w / w_{\max }$; (b) modal sensory voltage (upper surface). 


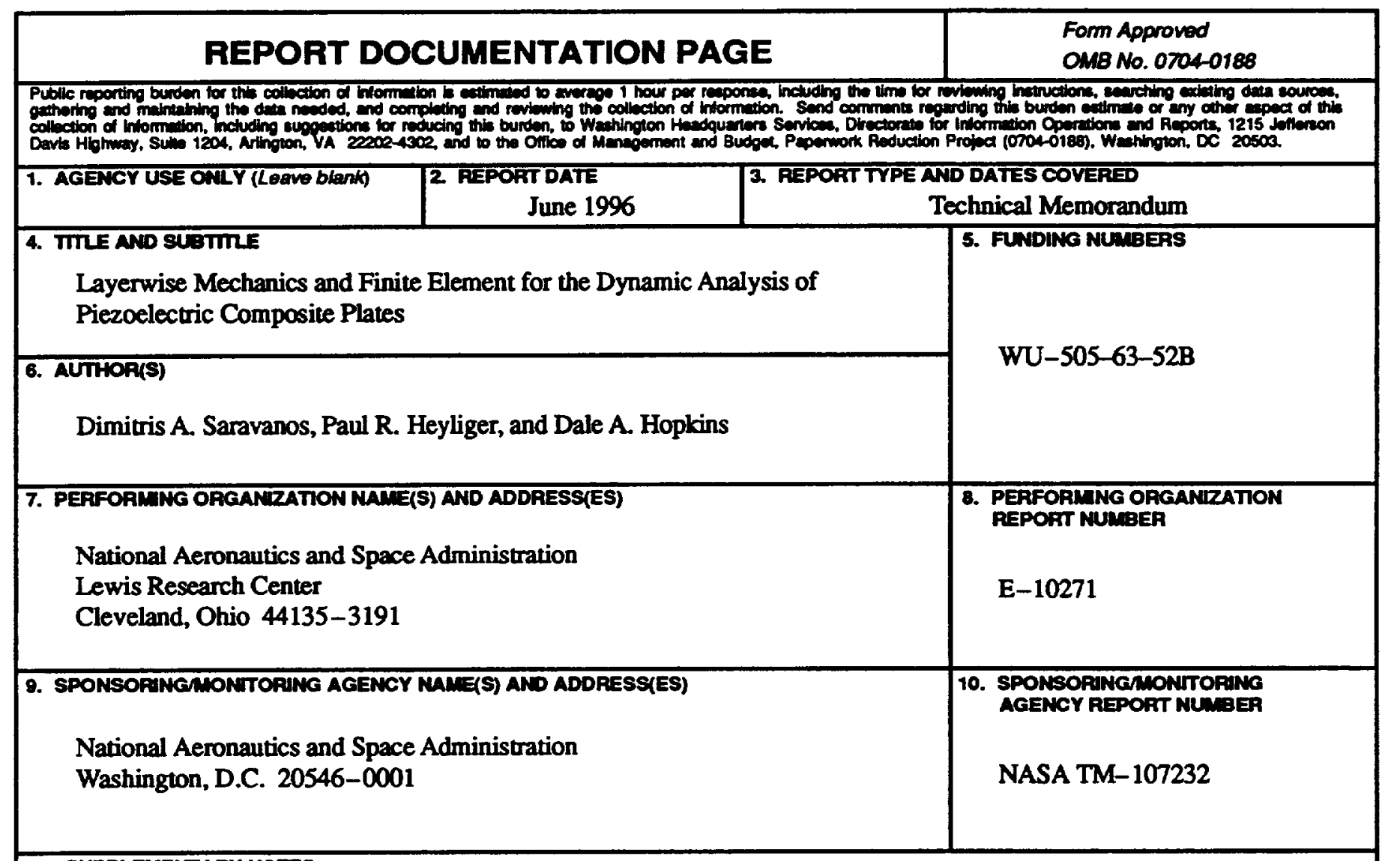

\section{SUPPLEMENTARY MOTES}

Dimitris A. Saravanos, Ohio Aerospace Institute, 22800 Cedar Point Road, Brook Park, Ohio 44142 (work funded by NASA Cooperative Agreement NCC3-391); Paul R. Heyliger, Colorado State University, Civil Engineering Department, Fort Collins, Colorado 80523-0002; and Dale A. Hop'ins, NASA Lewis Research Center. Responsible person, Dale A. Hopkins, organization code 5210, (216) 433-3260.

12a. DISTFIBUTONAVALABILTY STATEMENT 12b. DISTRIBUTION CODE

Unclassified - Unlimited

Subject Categories 39 and 24

This publication is available from the NASA Center for AeroSpace Information, (301) 621-0390.

13. ABSTRACT (Maximum 200 words)

Laminate and structural mechanics for the analysis of laminated composite plate structures with piezoelectric actuators and sensors are presented. The theories implement layerwise representations of displacements and electric potential, and can model both the global and local electromechanical response of smart composite laminates. Finite-element formulations are developed for the quasi-static and dynamic analysis of smart composite structures containing piezoelectric layers. Comparisons with an exact solution illustrate the accuracy, robustness and capability of the developed mechanics to capture the global and local response of thin and/or thick laminated piezoelectric plates. Additional correlations and numerical applications demonstrate the unique capabilities of the mechanics in analyzing the static and free-vibration response of composite plates with distributed piezoelectric actuators and sensors.

\section{SUBJECT TEAMS}

Composites; Laminates; Piezoelectricity; Sensors; Smart structures; Actuators; Structures; Finite element method; Plates; Plate theory

\begin{tabular}{|c|c|c|}
\hline $\begin{array}{c}\text { 17. SECUATY CLASSIFICATION } \\
\text { OF REPOFT } \\
\text { Unclassified }\end{array}$ & $\begin{array}{c}\text { 18. SECUATY CLASSIFICATION } \\
\text { OF THIS PAGE } \\
\text { Unclassified }\end{array}$ & $\begin{array}{c}\text { 19. SECUPTY CLASSIFICATION } \\
\text { OF ABSTIACT } \\
\text { Unclassified }\end{array}$
\end{tabular}




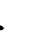




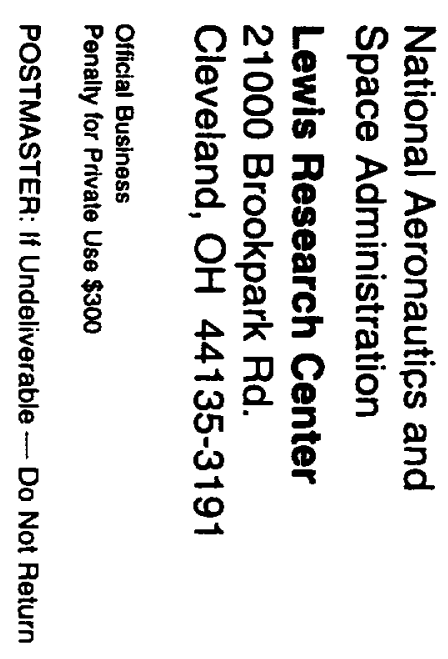

